\title{
Modulation of Intersystem Crossing Rate by Minor Ligand Modifications in Cyclometalated Platinum (II) Complexes
}

\author{
Marsel Z. Shafikov, ${ }^{*},{ }^{\ddagger}$ Dmitry N. Kozhevnikov, ${ }^{*}$ Michael Bodensteiner, ${ }^{\ddagger}$ Fabian Brandl, ${ }^{\S}$ and Rafa \\ Czerwieniec*,\& \\ ¥Ural Federal University, Mira 19, Ekaterinburg, 620002, Russia \\ $\dagger$ I. Postovsky Institute of Organic Synthesis, Ekaterinburg, 620041, Russia \\ $¥$ Center for Chemical Analysis, Faculty of Chemistry and Pharmacy, University Regensburg. \\ § Institut für Physikalische und Theoretische Chemie, Universität Regensburg, Universitätsstrasse 31, D- \\ 93053 Regensburg, Germany \\ *E-mail: shafikoff@gmail.com (M.Z.S), dnk.eburg@gmail.com (D.N.K.), rafal.czerwieniec@ur.de \\ (R.C.)
}

\section{Syntheses of the organic ligands HL1 - HL4.}

${ }^{1} \mathrm{H}$ NMR spectra were recorded on a Bruker AVANCE II NMR spectrometer operating at $400 \mathrm{MHz}$, and are referenced relative to residual ${ }^{1} \mathrm{H}$-solvent resonances.

\section{3-(4-bromophenlyl)-6-(4-fluorophenyl)-1,2,4-triazine.}

A mixture of commercially available 2-bromo-1-(4-fluorophenyl)-ethanone (7.56 $\mathrm{g}, 34.80 \mathrm{mmol})$, 4-bromobenzoic hydrazide (14.90 g, $69.00 \mathrm{mmol})$, and sodium acetate $(4.30 \mathrm{~g}, 52.50 \mathrm{mmol})$ was refluxed in a mixture of ethanol $(60 \mathrm{ml})$ and acetic acid $(20 \mathrm{ml})$ for 8 hours. Then, the mixture was cooled down to ambient temperature. The product precipitated as yellow crystals, which were filtered out, washed with ethanol, dried and used for the next step without further purification. Yield 77.5 \%. ${ }^{1} \mathrm{H}$ NMR $\left(\mathrm{CDCl}_{3}\right): \delta(\mathrm{ppm})=7.27\left(\mathrm{dd}, 2 \mathrm{H}, \mathrm{J}^{3}=8.69 \mathrm{~Hz}\right) ; 7.69(\mathrm{~d}$, $\left.2 \mathrm{H}, \mathrm{J}^{3}=8.60 \mathrm{~Hz}\right) ; 8.16\left(\mathrm{dd}, 2 \mathrm{H}, \mathrm{J}^{3}=8.69 \mathrm{~Hz}\right) ; 8.45\left(\mathrm{~d}, 2 \mathrm{H}, \mathrm{J}^{3}=8.60 \mathrm{~Hz}\right) ; 9.02(\mathrm{~s}$,

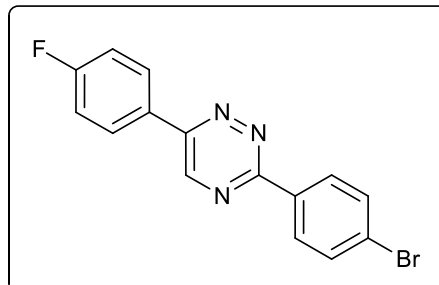
$1 \mathrm{H})$.

\section{3-(4-(5-ethylthien-2-yl)-phenyl)-6-(4-fluorophenyl)-1,2,4-triazine.}

A mixture of 3-(4-bromophenlyl)-6-(4-fluorophenyl)-1,2,4-triazine (1.47 g, $4.46 \mathrm{mmol}$ ) with 2-(tri-n-butyltin)-5-ethylthiophene (1.79 g, $4.46 \mathrm{mmol})$ was put into a Schlenk flask equipped with a magnetic stirrer and $30 \mathrm{ml}$ of dry DMF were added. Freeze-pump-thaw procedure was done three times and tetrakis-(triphenylphosphine)-palladium(0) (150.00 mg, 3\% mol) was quickly added into the flask under nitrogen atmosphere. The mixture was heated up to $100{ }^{\circ} \mathrm{C}$ and stirred at this temperature for 12 hours under nitrogen. Then, the solution was cooled down to ambient temperature, precipitation formed

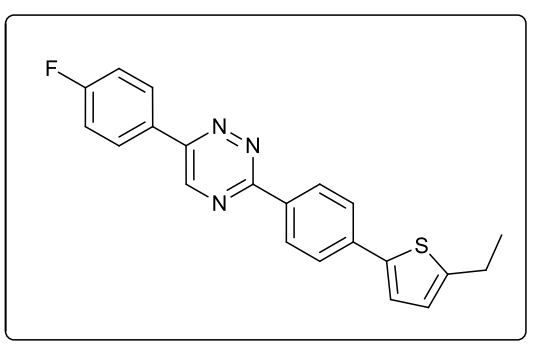
was filtered out, recrystallized from dimethylformamide (DMF) and dried. Yield 68.0 \%. ${ }^{1} \mathrm{H} \mathrm{NMR}\left(\mathrm{CDCl}_{3}\right): \delta$ $(\mathrm{ppm})=1.37\left(\mathrm{t}, 3 \mathrm{H}, \mathrm{J}^{3}=7.53 \mathrm{~Hz}\right) ; 2,90\left(\mathrm{qd}, 2 \mathrm{H}, \mathrm{J}^{3}=7.53 \mathrm{~Hz}, \mathrm{~J}^{4}=0.85 \mathrm{~Hz}\right) ; 6.82\left(\mathrm{dt}, 1 \mathrm{H}, \mathrm{J}^{3}=3.63 \mathrm{~Hz}, \mathrm{~J}^{4}=0.85 \mathrm{~Hz}\right)$; $7.26(\mathrm{~m}, 1 \mathrm{H}) ; 7.29\left(\mathrm{dd}, 2 \mathrm{H}, \mathrm{J}^{3}=6.49 \mathrm{~Hz}, \mathrm{~J}^{4}=2.94 \mathrm{~Hz}\right) ; 7.74\left(\mathrm{dt}, 2 \mathrm{H}, \mathrm{J}^{3}=8.70 \mathrm{~Hz}, \mathrm{~J}^{4}=1.91 \mathrm{~Hz}\right) ; 8.14-8.20(\mathrm{~m}, 2 \mathrm{H}) ; 8.56$ $\left(\mathrm{dt}, 2 \mathrm{H}, \mathrm{J}^{3}=8.70 \mathrm{~Hz}, \mathrm{~J}^{4}=1.91 \mathrm{~Hz}\right) ; 9.02(\mathrm{~s}, 1 \mathrm{H})$. 


\section{3-(thien-2-yl)-6-(4-fluorophenyl)-1,2,4-triazine.}

A mixture of 2-bromo-1-(4-fluorophenyl)-ethanone (7.23 g, $33.00 \mathrm{mmol})$, 2thienoylhydrazide $(9.37 \mathrm{~g}, 66.00 \mathrm{mmol})$ and sodium acetate $(4.00 \mathrm{~g}, 48.78 \mathrm{mmol})$ was refluxed in a mixture of ethanol $(60 \mathrm{ml})$ and acetic acid $(20 \mathrm{ml})$ for 8 hours and was cooled down to ambient temperature. The precipitated crystals of product were filtered out, washed with ethanol, dried under reduced pressure and used for the next step without further purification. Yield 43.2\%. ${ }^{1} \mathrm{H} \mathrm{NMR}\left(\mathrm{CDCl}_{3}\right): \delta(\mathrm{ppm})=7.20-$ $7.30(\mathrm{~m}, 3 \mathrm{H}) ; 7.60\left(\mathrm{dd}, 1 \mathrm{H}, \mathrm{J}^{3}=5.00 \mathrm{~Hz}, \mathrm{~J}^{4}=1.22 \mathrm{~Hz}\right) ; 8.09-8.15(\mathrm{~m}, 2 \mathrm{H}) ; 8.17(\mathrm{dd}$,

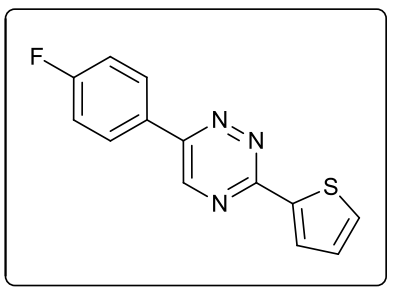
$\left.1 \mathrm{H}, \mathrm{J}^{3}=2.74 \mathrm{~Hz}, \mathrm{~J}^{4}=1.22 \mathrm{~Hz}\right) ; 8.93(\mathrm{~s}, 1 \mathrm{H})$.

\section{3-(5-bromothien-2-yl)-6-(4-fluorophenyl)-1,2,4-triazine.}

A mixture of 3-(thien-2-yl)-6-(4-fluorophenyl)-1,2,4-triazine $(2.57 \mathrm{~g}, 10.00 \mathrm{mmol})$ with N-bromosuccinimide (NBS) $(1.87 \mathrm{~g}, 10.50 \mathrm{mmol})$ was kept at $100{ }^{\circ} \mathrm{C}$ in a flask with $65 \mathrm{ml}$ of DMF for 5 hours. Then water was added and a precipitate appeared. The precipitate was filtered out, washed with cold ethanol, dried and used for the next step without further purification. Yield 85.0\%. ${ }^{1} \mathrm{H} \mathrm{NMR}\left(\mathrm{CDCl}_{3}\right)$ : $\delta(\mathrm{ppm})=7.20\left(\mathrm{~d}, 1 \mathrm{H}, \mathrm{J}^{3}=3.98 \mathrm{~Hz}\right) ; 7.25-7.31(\mathrm{~m}, 2 \mathrm{H}) ; 7.93\left(\mathrm{~d}, 1 \mathrm{H}, \mathrm{J}^{3}=3.98 \mathrm{~Hz}\right) ; 8.10-$

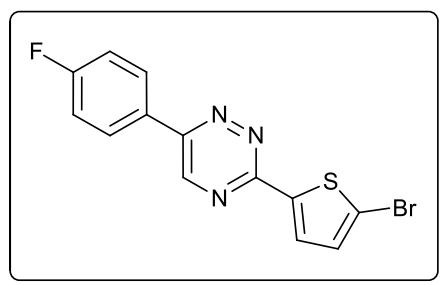
$8.18(\mathrm{~m}, 2 \mathrm{H}) ; 8.93(\mathrm{~s}, 1 \mathrm{H})$.

\section{3-(5-(5-ethylthien-2-yl)-thien-2-yl)-6-(4-fluorophenyl)-1,2,4-triazine.}

A mixture of 3-(5-bromothien-2-yl)-6-(4-fluorophenyl)-1,2,4-triazine $(1.50 \mathrm{~g}, 4.00 \mathrm{mmol})$ with 2-(tri-n-butyltin)-5-ethylthiophene $(1.79 \mathrm{~g}, 4.46$ mmol) was put in a Schlenk flask equipped with a magnetic stirrer and 30 $\mathrm{ml}$ of dry DMF were added. Freeze-pump-thaw procedure was done three times and tetrakis(triphenylphosphine)palladium(0) (150.00 mg, 3\% mol) was quickly added to the flask. The mixture was warmed up to $100{ }^{\circ} \mathrm{C}$ and kept at it for 12 hours under nitrogen. Then solution was cooled down and

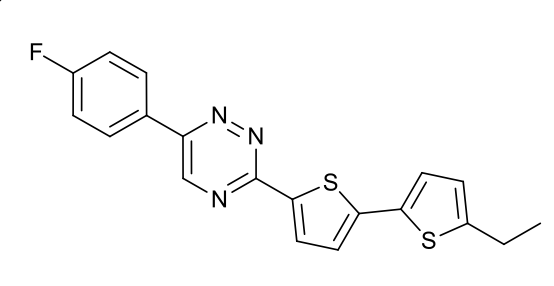
precipitate formed afterward was filtered out, recrystallized from acetic acid and dried. Yield 80.0 \% ${ }^{1} \mathrm{H}$ NMR $\left(\mathrm{CDCl}_{3}\right): \delta(\mathrm{ppm})=1.35\left(\mathrm{t}, 3 \mathrm{H}, \mathrm{J}^{3}=7.53 \mathrm{~Hz}\right) ; 2.87\left(\mathrm{qd}, 2 \mathrm{H}, \mathrm{J}^{3}=7.53 \mathrm{~Hz}, \mathrm{~J}^{4}=0.99 \mathrm{~Hz}\right) ; 6.76\left(\mathrm{dt}, 1 \mathrm{H}, \mathrm{J}^{3}=3.61\right.$ $\left.\mathrm{Hz}, \mathrm{J}^{4}=0.99 \mathrm{~Hz}\right) ; 7.16\left(\mathrm{~d}, 1 \mathrm{H}, \mathrm{J}^{3}=3,61 \mathrm{~Hz}\right) ; 7.20\left(\mathrm{~d}, 1 \mathrm{H}, \mathrm{J}^{3}=3.95 \mathrm{~Hz}\right) ; 7.22-7.29(\mathrm{~m}, 2 \mathrm{H}) ; 8.06\left(\mathrm{~d}, 1 \mathrm{H}, \mathrm{J}^{3}=\right.$ $3.95 \mathrm{~Hz}) ; 8.09-8.15(\mathrm{~m}, 2 \mathrm{H}) ; 8.89(\mathrm{~s}, 1 \mathrm{H})$.

\section{2-(5-ethylthien-2-yl)-phenyl)-5-(4-fluorophenyl)-pyridine.}

To 3-(4-(5-ethylthien-2-yl)-phenyl)-6-(4-fluorophenyl)-1,2,4-triazine (750 $\mathrm{mg}, 2.07 \mathrm{mmol})$ in an autoclave equipped with magnetic stirrer, 5 equivalents of 2,5-norbornadiene $(0.955 \mathrm{~g}, 10.39 \mathrm{mmol})$ and $10 \mathrm{ml}$ of oxylene were added. Autoclave was closed tightly and left at $200{ }^{\circ} \mathrm{C}$ in an oil bath for overnight. The solvent was evaporated at reduced pressure and 10 $\mathrm{ml}$ of methanol were added to the oily residue and flask was put in a freezer for an hour. Precipitated product was filtered out, washed with methanol and dried. Yield $53 \%$. M.p. $=134.0{ }^{\circ} \mathrm{C} .{ }^{1} \mathrm{H}$ NMR $\left(\mathrm{CDCl}_{3}\right): \delta(\mathrm{ppm})=1.36$

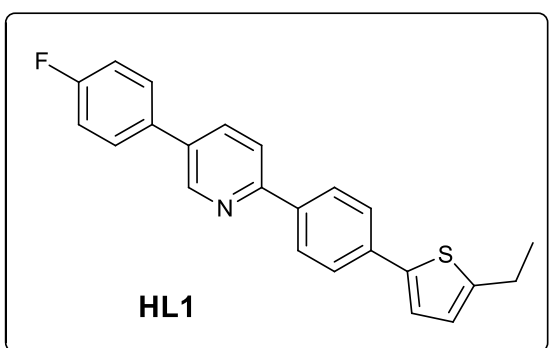
$\left(\mathrm{t}, 3 \mathrm{H}, \mathrm{J}^{3}=7.55 \mathrm{~Hz}\right) ; 2.88\left(\mathrm{q}, 2 \mathrm{H}, \mathrm{J}^{3}=7.55 \mathrm{~Hz}\right) ; 6.79\left(\mathrm{~d}, 1 \mathrm{H}, \mathrm{J}^{3}=3.49 \mathrm{~Hz}\right) ; 7.19\left(\mathrm{t}, 2 \mathrm{H}, \mathrm{J}^{3}=8.59 \mathrm{~Hz}\right) ; 7.22(\mathrm{~d}$, $\left.1 \mathrm{H}, \mathrm{J}^{3}=3.54 \mathrm{~Hz}\right) ; 7.60\left(\mathrm{dd}, 2 \mathrm{H}, \mathrm{J}^{3}=8.70 \mathrm{~Hz}, \mathrm{~J}^{4}=5.28 \mathrm{~Hz}\right) ; 7.68\left(\mathrm{~d}, 2 \mathrm{H}, \mathrm{J}^{3}=8.37 \mathrm{~Hz}\right) ; 7.81\left(\mathrm{~d}, 1 \mathrm{H}, \mathrm{J}^{3}=8.70\right.$ $\mathrm{Hz}) ; 7.9\left(\mathrm{dd}, 1 \mathrm{H}, \mathrm{J}^{3}=8.22 \mathrm{~Hz}, \mathrm{~J}^{4}=2.39 \mathrm{~Hz}\right) ; 8.05\left(\mathrm{~d}, 2 \mathrm{H}, \mathrm{J}^{3}=8.37 \mathrm{~Hz}\right) ; 8.9\left(\mathrm{~d}, 1 \mathrm{H}, \mathrm{J}^{4}=1.03 \mathrm{~Hz}\right)$. Found, \%: C, 76.78; H, 4.98; N, 3.95; S, 8.89. Calculated, \%: C, 76.85; H, 5.05; N, 3.90; S, 8.92. 


\section{2-(5-ethylthien-2-yl)-pnenyl)-5-(4-fluorophenyl)-c-cyclopentene-pyridine.}

To 3-(4-(5-ethylthien-2-yl)phenyl)-6-(4-fluorophenyl)-1,2,4-triazine (750 $\mathrm{mg}, 2.07 \mathrm{mmol})$ in round bottom flask equipped with a magnetic stirrer 3 equivalents of 1-(4-morpholino)-cyclopentene $(950 \mathrm{mg}, 6.21 \mathrm{mmol})$ were added. Flask was heated up to $180^{\circ} \mathrm{C}$ in an oil bath and kept at it for 1 hour. Then 2 more equivalents of 1-(4-morpholino)-cyclopentene (633 mg, 4.14 mmol) were added and flask was kept at $180^{\circ} \mathrm{C}$ for 1 additional hour. $10 \mathrm{ml}$ of methanol were added to the cooled down oily mixture and flask was put in freezer. Precipitated product was filtered out, washed with methanol and

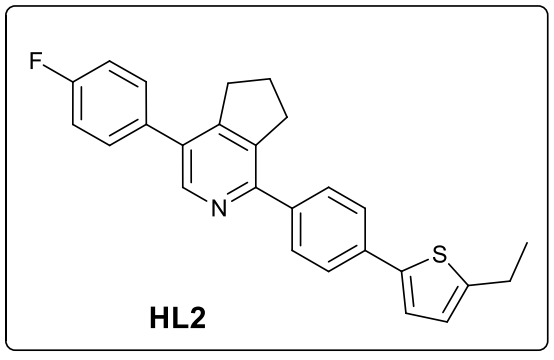
dried. Yield $67 \%$. M.p. $=182.4{ }^{\circ} \mathrm{C} .{ }^{1} \mathrm{H}$ NMR $\left(\mathrm{CDCl}_{3}\right): \delta(\mathrm{ppm})=1.35\left(\mathrm{t}, 3 \mathrm{H}, \mathrm{J}^{3}=7.51 \mathrm{~Hz}\right) ; 2.09\left(\mathrm{~m}, 2 \mathrm{H}, \mathrm{J}^{3}=\right.$ 7.34) $2.88\left(\mathrm{q}, 2 \mathrm{H}, \mathrm{J}^{3}=7.51 \mathrm{~Hz}\right) ; 3.02\left(\mathrm{t}, 2 \mathrm{H}, \mathrm{J}^{3}=7.34\right) ; 3.20\left(\mathrm{t}, 2 \mathrm{H}, \mathrm{J}^{3}=7.34 \mathrm{~Hz}\right) ; 6.75\left(\mathrm{~d}, 1 \mathrm{H}, \mathrm{J}^{3}=3.56 \mathrm{~Hz}\right)$; $7.17\left(\mathrm{t}, 2 \mathrm{H}, \mathrm{J}^{3}=8.68 \mathrm{~Hz}\right) ; 7.21\left(\mathrm{~d}, 1 \mathrm{H}, \mathrm{J}^{3}=3.56 \mathrm{~Hz}\right) ; 7.46\left(\mathrm{dd}, 2 \mathrm{H}, \mathrm{J}^{3}=8.70 \mathrm{~Hz}, \mathrm{~J}^{3}=5.34 \mathrm{~Hz}\right) ; 7.67\left(\mathrm{~d}, 2 \mathrm{H}, \mathrm{J}^{3}\right.$ $=8.37 \mathrm{~Hz}) ; 7.81\left(\mathrm{~d}, 2 \mathrm{H}, \mathrm{J}^{3}=8,34 \mathrm{~Hz}\right) ; 8.52(\mathrm{~s}, 1 \mathrm{H})$. Found, \%: C, 78.11; H, 5.62; N, 3.68; S, 7.99; F, 4.55. Calculated, \%: C, 78.16; H, 5.55; N, 3.51; S, 8.03; F, 4.76.

\section{2-(5-(5-ethylthien-2-yl)-thien-2-yl)-5-(4-fluorophenyl)-pyridine}

To 3-(5-(5-ethylthien-2-yl)-thien-2yl)-6-(4-fluorophenyl)-1,2,4-triazine $(500 \mathrm{mg}, 1.36 \mathrm{mmol})$ in an autoclave equipped with a magnetic stirrer, 5 equivalents of 2,5-norbornadine $(0.626 \mathrm{~g}, 6.81 \mathrm{mmol})$ and $10 \mathrm{ml}$ of oxylene were added. Autoclave was closed tightly and left at $200{ }^{\circ} \mathrm{C}$ in an oil bath for overnight. The solvent was evaporated at reduced pressure and $10 \mathrm{ml}$ of methanol were added to the oily residue and the flask was put in a freezer. Precipitated product was filtered out, washed with

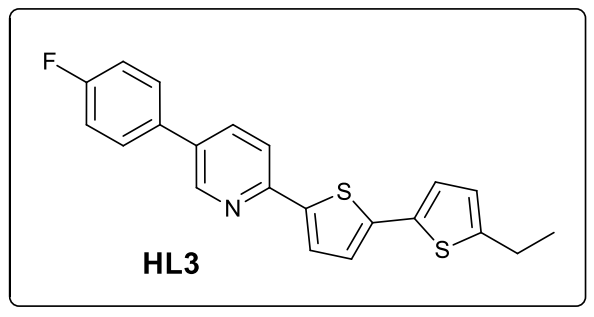
methanol and dried. Yield $55 \%$. M.p. $=110.0{ }^{\circ} \mathrm{C} .{ }^{1} \mathrm{H}$ NMR $\left(\mathrm{CDCl}_{3}\right): \delta(\mathrm{ppm})=1.34\left(\mathrm{t}, 3 \mathrm{H}, \mathrm{J}^{3}=7.69 \mathrm{~Hz}\right) ; 2.86$ $\left(\mathrm{q}, 2 \mathrm{H}, \mathrm{J}^{3}=7.69 \mathrm{~Hz}\right) ; 6.73\left(\mathrm{~d}, 1 \mathrm{H}, \mathrm{J}^{3}=3.59 \mathrm{~Hz}\right) ; 7.09\left(\mathrm{~d}, 1 \mathrm{H}, \mathrm{J}^{3}=3.55 \mathrm{~Hz}\right) ; 7.12\left(\mathrm{~d}, 1 \mathrm{H}, \mathrm{J}^{3}=3.85 \mathrm{~Hz}\right) ; 7.18(\mathrm{t}$, $\left.2 \mathrm{H}, \mathrm{J}^{3}=8.63 \mathrm{~Hz}\right) ; 7.49\left(\mathrm{~d}, 1 \mathrm{H}, \mathrm{J}^{3}=3.84 \mathrm{~Hz}\right) ; 7.56\left(\mathrm{dd}, 2 \mathrm{H}, \mathrm{J}^{3}=8.70 \mathrm{~Hz}, \mathrm{~J}^{3}=5.24 \mathrm{~Hz}\right) ; 7,69\left(\mathrm{~d}, 1 \mathrm{H}, \mathrm{J}^{3}=8.70\right)$; $7.83\left(\mathrm{dd}, 1 \mathrm{H}, \mathrm{J}^{3}=8.28, \mathrm{~J}^{4}=2.36\right) ; 8.75\left(\mathrm{~d}, 1 \mathrm{H}, \mathrm{J}^{4}=1.11 \mathrm{~Hz}\right)$. Found, \%: C, 69.14; H, 4.42; N, 3.84; S, 17.55. Calculated, \%: C, 69.01; H, 4.41; N, 3.83; S, 17.45 .

\section{2-(5-(5-ethylthien-2-yl)-thien-2-yl)-5-(4-fluorophenyl)-c-cyclopentene-pyridine.}

To 3-(5-(5-ethylthien-2-yl)-thien-2yl)-6-(4-fluorophenyl)-1,2,4-triazine (5) $(504 \mathrm{mg}, 1.37 \mathrm{mmol})$ in round bottom flask equipped with magnetic stirrer 3 equivalents of 1-(4-morpholino)cyclopentene $(650 \mathrm{mg}, 4.11$ mmol) were added. Flask was heated up to $180{ }^{\circ} \mathrm{C}$ in an oil bath and kept at it for 1 hour. Then 2 more equivalents of 1-(4-morpholino)cyclopentene (436 mg, $2.74 \mathrm{mmol}$ ) were added and the flask was kept at $180{ }^{\circ} \mathrm{C}$ for 1 additional hour. $10 \mathrm{ml}$ of methanol were added to the cooled down oily

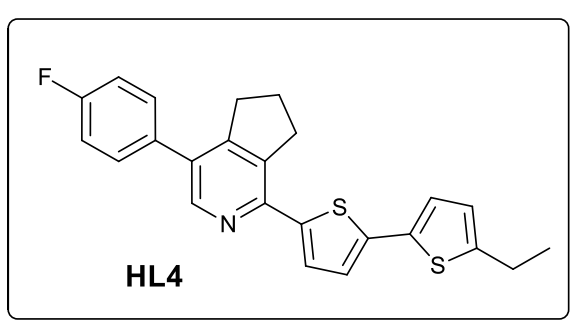
mixture and the flask was put in a freezer for product to precipitate.

Precipitated product was filtered out, washed with methanol and dried. Yield $50 \%$. M.p. $=136.2{ }^{\circ} \mathrm{C} .{ }^{1} \mathrm{H}$ NMR $\left(\mathrm{CDCl}_{3}\right): \delta(\mathrm{ppm})=1.33\left(\mathrm{t}, 3 \mathrm{H}, \mathrm{J}^{3}=7.53 \mathrm{~Hz}\right) ; 2.17\left(\mathrm{~m}, 2 \mathrm{H}, \mathrm{J}^{3}=7.45\right) 2.85\left(\mathrm{q}, 2 \mathrm{H}, \mathrm{J}^{3}=7.53 \mathrm{~Hz}\right) ; 3.01\left(\mathrm{t}, 2 \mathrm{H}, \mathrm{J}^{3}\right.$ $=7.45 \mathrm{~Hz}) ; 3.23\left(\mathrm{t}, 2 \mathrm{H}, \mathrm{J}^{3}=7.45 \mathrm{~Hz}\right) ; 6.72\left(\mathrm{~d}, 1 \mathrm{H}, \mathrm{J}^{3}=3.57 \mathrm{~Hz}\right) ; 7.09\left(\mathrm{~d}, 1 \mathrm{H}, \mathrm{J}^{3}=3.57 \mathrm{~Hz}\right) ; 7.15(\mathrm{~m}, 3 \mathrm{H}) ; 7.38$ $\left(\mathrm{d}, 1 \mathrm{H}, \mathrm{J}^{3}=3.92\right) ; 7.42\left(\mathrm{dd}, 2 \mathrm{H}, \mathrm{J}^{3}=8.61, \mathrm{~J}^{4}=5.33 \mathrm{~Hz}\right) ; 8.39(\mathrm{~s}, 1 \mathrm{H})$. Found, \%: C, 71.17; H, 4.87; N, 3.49; $\mathrm{S}$, 15.81; F, 4.59. Calculated, \%: C, 71.08; H, 4.97; N, 3.45; S, 15.81; F, 4.68. 


\section{Syntheses of the platinum complexes $1-4$.}

General procedure: A ligand HL1-HL4 was dissolved in acetic acid in a round bottom flask equipped with magnetic stirrer and heated up to $80^{\circ} \mathrm{C}$. To the resulted solution one equivalent of tetrachloropotassiumplatinat (II) $\left(\mathrm{K}_{2} \mathrm{PtCl}_{4}\right)$, preliminarily dissolved in the smallest possible amount of water, was added dropwise and the resulting mixture was refluxed for 24 hours at $120^{\circ} \mathrm{C}$. Then, the reaction mixture was cooled down to ambient temperature. A green precipitate was filtered out, washed with methanol and dried. The thus obtained chlorobridged dimeric complex $\left[\mathrm{Pt}_{2}(\mu-\mathrm{Cl})_{2}(\mathrm{~L} 1-\mathrm{L} 4)_{2}\right]$ was dissolved in acetone and 10 equivalents of sodium acetylacetonate $(\mathrm{Na}(\mathrm{acac}))$ were added. The solution was refluxed for 48 hours. Then, the solvent was evaporated at reduced pressure, $70 \mathrm{ml}$ of water were added to the flask and product was extracted with dichloromethane. Resulted solution was dried under anhydrous sodium sulphate and solvent was evaporated under reduced pressure. Crude product was purified by column chromatography (silica gel, dichloromethane).

\section{Complex 1, [Pt(L1)(acac)].}

Yield $=77 \%$. M.p. $=270.3{ }^{\circ} \mathrm{C} .{ }^{1} \mathrm{H}$ NMR $\left(\mathrm{CDCl}_{3}\right): \delta(\mathrm{ppm})=1.28(\mathrm{t}, 3 \mathrm{H}$, $\left.\mathrm{J}^{3}=7.51 \mathrm{~Hz}\right) ; 2.01(\mathrm{~s}, 3 \mathrm{H}) ; 2.05(\mathrm{~s}, 3 \mathrm{H}) ; 2.89\left(\mathrm{q}, 2 \mathrm{H}, \mathrm{J}^{3}=7.51 \mathrm{~Hz}\right) ; 5.48$ $(\mathrm{s}, 1 \mathrm{H}) ; 6.77\left(\mathrm{~d}, 1 \mathrm{H}, \mathrm{J}^{3}=3.22 \mathrm{~Hz}\right) ; 7.18\left(\mathrm{t}, 2 \mathrm{H}, \mathrm{J}^{3}=8.51 \mathrm{~Hz}\right) ; 7.23(\mathrm{~d}, 1 \mathrm{H}$, $\left.\mathrm{J}^{3}=3.47 \mathrm{~Hz}\right) ; 7.30\left(\mathrm{dd}, 1 \mathrm{H}, \mathrm{J}^{3}=8.02 \mathrm{~Hz}, \mathrm{~J}^{4}=1.26 \mathrm{~Hz}\right) ; 7.37\left(\mathrm{~d}, 1 \mathrm{H}, \mathrm{J}^{3}=\right.$ $8.02 \mathrm{~Hz}) ; 7.55(\mathrm{~m}, 3 \mathrm{H}) ; 7.81(\mathrm{~d}, 1 \mathrm{H}, 1.2 \mathrm{~Hz}) ; 7.91\left(\mathrm{dd}, 1 \mathrm{H}, \mathrm{J}^{3}=8.37 \mathrm{~Hz}\right.$, $\left.\mathrm{J}^{4}=1.94 \mathrm{~Hz}\right)$; 9.17 (s, 1H). Found, \%: C, 51.26; H, 3.68; N, 2.21; S, 4.92 . Calculated, \%: C, 51.45; H, 3.86; N, 2.14; S, 4.91.

\section{Complex 2, Pt(L2)(acac).}

Yield 82\%. M.p. $=297.6{ }^{\circ} \mathrm{C} .{ }^{1} \mathrm{H}$ NMR $\left(\mathrm{CDCl}_{3}\right): \delta(\mathrm{ppm})=1.28(\mathrm{t}, 3 \mathrm{H}$, $\left.\mathrm{J}^{3}=7.54 \mathrm{~Hz}\right) ; 1.87(\mathrm{~s}, 3 \mathrm{H}) ; 1.96(\mathrm{~s}, 3 \mathrm{H}) 2.09(\mathrm{~m}, 2 \mathrm{H}) 2.81\left(\mathrm{qd}, 2 \mathrm{H}, \mathrm{J}^{3}=\right.$ $\left.7.54 \mathrm{~Hz}, \mathrm{~J}^{4}=0.83 \mathrm{~Hz}\right) ; 2.88\left(\mathrm{t}, 2 \mathrm{H}, \mathrm{J}^{3}=7.50 \mathrm{~Hz}\right) ; 3.28\left(\mathrm{t}, 2 \mathrm{H}, \mathrm{J}^{3}=7.50\right.$ $\mathrm{Hz}) ; 5.39(\mathrm{~s}, 1 \mathrm{H}) ; 6.70\left(\mathrm{~d}, 1 \mathrm{H}, \mathrm{J}^{3}=3.56 \mathrm{~Hz}\right) ; 7.08\left(\mathrm{t}, 2 \mathrm{H}, \mathrm{J}^{3}=8.70 \mathrm{~Hz}\right)$; $7.15\left(\mathrm{~d}, 1 \mathrm{H}, \mathrm{J}^{3}=3.55 \mathrm{~Hz}\right) ; 7.20\left(\mathrm{dd}, 1 \mathrm{H}, \mathrm{J}^{3}=8.10 \mathrm{~Hz}, \mathrm{~J}^{4}=1.98 \mathrm{~Hz}\right) ; 7.35$ $\left(\mathrm{dd}, 2 \mathrm{H}, \mathrm{J}^{3}=8.80 \mathrm{~Hz}, \mathrm{~J}^{3}=5.30 \mathrm{~Hz}\right) ; 7.39\left(\mathrm{~d}, 1 \mathrm{H}, \mathrm{J}^{3}=8.23 \mathrm{~Hz}\right) ; 7.75(\mathrm{~d}$, $\left.1 \mathrm{H}, \mathrm{J}^{3}=1.95 \mathrm{~Hz}\right) ; 8.73$ (s, 1H). Found, \%: C, 53.44; H, 4.20; N, 2.08. Calculated, \%: C, 53.67; H, 4.21; N, 2.02.

\section{Complex 3, [Pt(L3)(acac)].}

Yield $=86 \%$. M.p. $=267,6{ }^{\circ} \mathrm{C} .{ }^{1} \mathrm{H}$ NMR $\left(\mathrm{CDCl}_{3}\right): \delta(\mathrm{ppm})=1,36\left(\mathrm{t}, 3 \mathrm{H}, \mathrm{J}^{3}\right.$ $=7.43 \mathrm{~Hz}) ; 2.00(\mathrm{~s}, 6 \mathrm{H}) 2.87\left(\mathrm{q}, 2 \mathrm{H}, \mathrm{J}^{3}=7.43 \mathrm{~Hz}\right) ; 5.49(\mathrm{~s}, 1 \mathrm{H}) ; 6.73(\mathrm{~d}$, $\left.1 \mathrm{H}, \mathrm{J}^{3}=1.96 \mathrm{~Hz}\right) ; 7.08\left(\mathrm{~d}, 1 \mathrm{H}, \mathrm{J}^{3}=2.82 \mathrm{~Hz}\right) ; 7.12-7.30(\mathrm{~m}, 4 \mathrm{H}) ; 7.52(\mathrm{dd}$, $\left.2 \mathrm{H} \mathrm{J}^{3}=7.60 \mathrm{~Hz}, \mathrm{~J}^{3}=5.41 \mathrm{~Hz}\right) ; 7.76\left(\mathrm{~d}, 1 \mathrm{H} \mathrm{J}^{3}=8.08 \mathrm{~Hz}\right) ; 8.97(\mathrm{~s}, 1 \mathrm{H})$. Found, \%: C, 47.51; H, 3.38; N, 2.16; S, 9.70. Calculated, \%: C, 47.34; H, $3.51 ; \mathrm{N}, 2,12 ; \mathrm{S}, 9.72$.

\section{Complex 4, [Pt(L4)(acac)].}

Yield 73\%. M.p. $=301.5{ }^{\circ} \mathrm{C} .{ }^{1} \mathrm{H}$ NMR $\left(\mathrm{CDCl}_{3}\right): \delta(\mathrm{ppm})=1.26\left(\mathrm{t}, 3 \mathrm{H}, \mathrm{J}^{3}=\right.$ $7.55 \mathrm{~Hz}) ; 1.87(\mathrm{~s}, 3 \mathrm{H}) ; 1.92(\mathrm{~s}, 3 \mathrm{H}) ; 2.08(\mathrm{~m}, 2 \mathrm{H}) 2.77$ (q, 2H, ${ }^{3}=7.55$ $\mathrm{Hz}) ; 2.92\left(\mathrm{t}, 2 \mathrm{H}, \mathrm{J}^{3}=7.54 \mathrm{~Hz}\right) ; 3.10\left(\mathrm{t}, 2 \mathrm{H}, \mathrm{J}^{3}=7.54 \mathrm{~Hz}\right) ; 5.39(\mathrm{~s}, 1 \mathrm{H}) ; 6.63$ $\left(\mathrm{d}, 1 \mathrm{H}, \mathrm{J}^{3}=3.54 \mathrm{~Hz}\right) ; 6.96\left(\mathrm{~d}, 1 \mathrm{H}, \mathrm{J}^{3}=3.49 \mathrm{~Hz}\right) ; 7.03-7.13(\mathrm{~m}, 3 \mathrm{H}) ; 7.34$ $\left(\mathrm{dd}, 2 \mathrm{H}, \mathrm{J}^{3}=8.69 \mathrm{~Hz}, \mathrm{~J}^{4}=5.33 \mathrm{~Hz}\right) ; 8.48(\mathrm{~s}, 1 \mathrm{H})$. Found, \%: C, 49.68; H, 3.76; N, 2.05; S, 9.06. Calculated, \%: C, 49.78; H, 3.89; N, 2.00; S, 9.16.
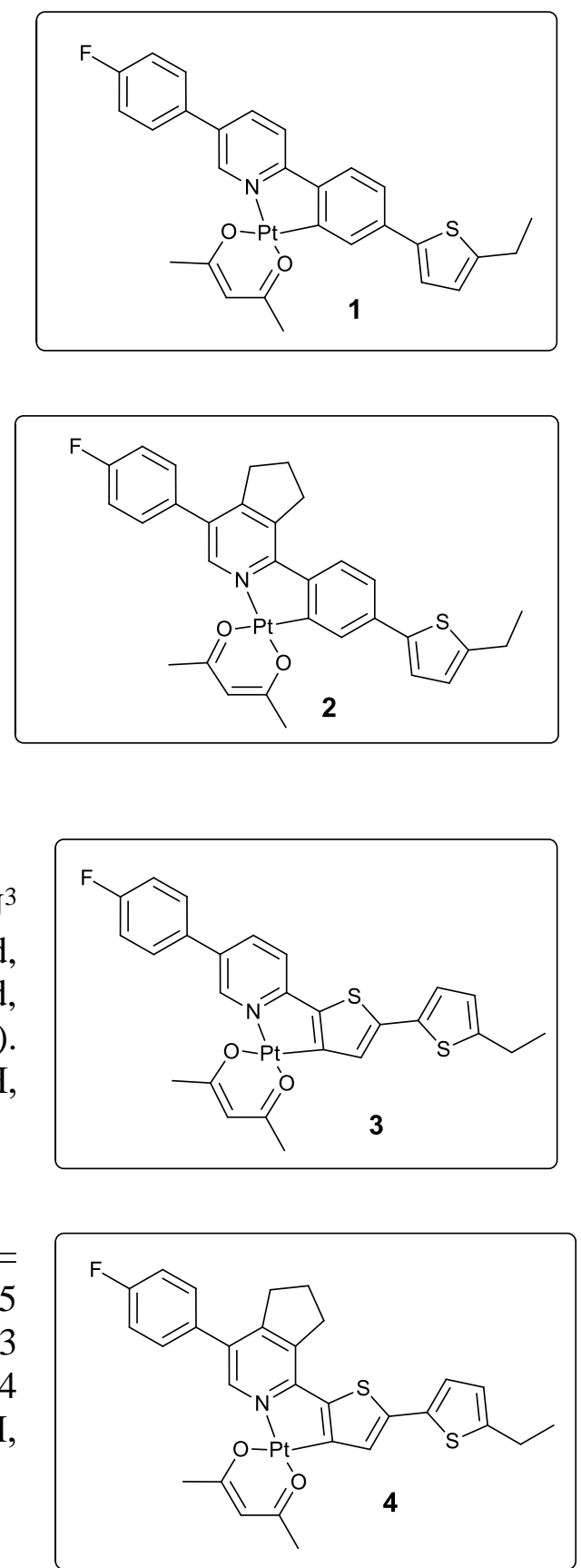


\section{X-ray diffraction studies.}

A clear brown needle-shaped crystal of $\mathbf{3}$ and a brown plate-shaped crystal of $\mathbf{4}$ were selected and mounted on a MITIGEN holder with inert oil on an Agilent Technologies SuperNova Atlas S2 CCD (3) and an Oxford Diffraction Xcalibur Gemini Ultra Atlas CCD (4) diffractometer, respectively. Data collection, cell refinement and data reduction were performed with CrysAlisPro in different versions. ${ }^{1}$ Using Olex $2^{2}$, the structures were solved with ShelXS or ShelXT and refined with ShelXL (version 2014/7) ${ }^{3}$.

For the model of $\mathbf{3}$ distance and displacement parameters were used for the refinement of the disordered minor position (Figure S1). The crystals of $\mathbf{4}$ were of poor quality with a very high mosaicity. The chosen crystal turned out to be an inversion twin with a ratio of 1:1 within the standard deviations. However, a change to space group Pnma leads to additional disorder of the $-\mathrm{C}_{6} \mathrm{H}_{4} \mathrm{~F}$ substituent. For reason of the poor quality, several ISOR restraints were applied. In addition, the residual density is high, but located nearby the platinum atom in less than $1 \AA$ distance.

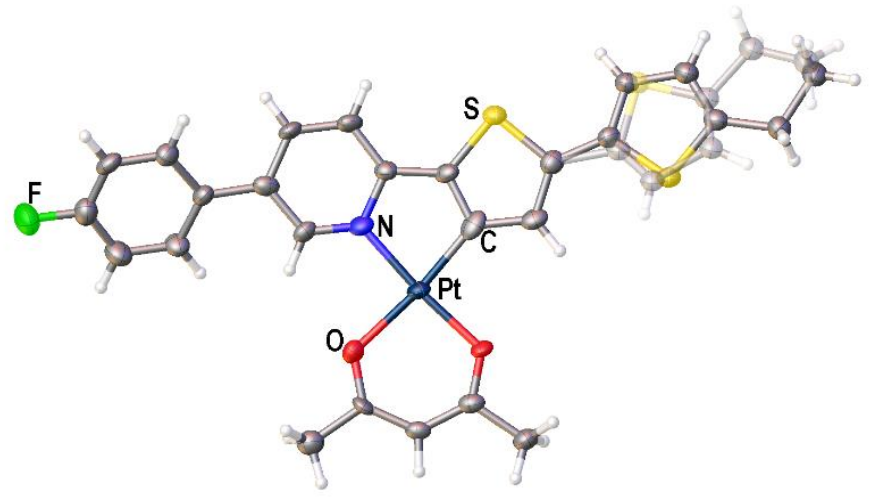

Figure S1. Disorder of the ethyl thiophene substituent in $\mathbf{3}$ (17.6 per cent minor component shown translucent). 
Table S1. Crystal data, data collection, and structure refinement details.

\begin{tabular}{|c|c|c|}
\hline Compound & 3 & 4 \\
\hline Formula & $\mathrm{C}_{26} \mathrm{H}_{22} \mathrm{FNO}_{2} \mathrm{PtS}_{2}$ & $\mathrm{C}_{29} \mathrm{H}_{26} \mathrm{FNO}_{2} \mathrm{PtS}_{2}$ \\
\hline$D_{\text {calc. }} / \mathrm{g} \mathrm{cm}^{-3}$ & 1.892 & 1.825 \\
\hline$\mu / \mathrm{mm}^{-1}$ & 13.314 & 12.150 \\
\hline Formula Weight & 658.65 & 698.72 \\
\hline Colour & clear brown & brown \\
\hline Shape & needle & plate \\
\hline Max Size/mm & 0.42 & 0.10 \\
\hline Mid Size/mm & 0.09 & 0.03 \\
\hline Min Size/mm & 0.07 & 0.02 \\
\hline$T / \mathrm{K}$ & $123.01(10)$ & $123.00(14)$ \\
\hline Crystal System & monoclinic & orthorhombic \\
\hline Flack Parameter & $-0.036(14)$ & $0.0(12)$ \\
\hline Hooft Parameter & $-0.021(12)$ & $0.490(14)$ \\
\hline Space Group & $\mathrm{P} 2_{1}$ & $\mathrm{Pca} 2{ }_{1}$ \\
\hline$a / \AA$ & $8.9809(4)$ & $20.6742(5)$ \\
\hline$b / \AA$ & $11.5439(3)$ & $17.3852(4)$ \\
\hline$c / \AA$ & $11.9873(5)$ & $7.07539(16)$ \\
\hline$\alpha l^{\circ}$ & 90 & 90 \\
\hline$\beta l^{\circ}$ & $111.512(5)$ & 90 \\
\hline$\gamma 1^{\circ}$ & 90 & 90 \\
\hline $\mathrm{V} / \AA^{3}$ & $1156.20(8)$ & $2543.06(10)$ \\
\hline$Z$ & 2 & 4 \\
\hline$Z^{\prime}$ & 1 & 1 \\
\hline$\Theta_{\min } l^{\circ}$ & 3.964 & 3.321 \\
\hline$\Theta_{\max } 1^{\circ}$ & 70.458 & 66.886 \\
\hline Measured Refl. & 4569 & 21311 \\
\hline Independent Refl. & 3076 & 4379 \\
\hline Reflections Used & 3003 & 3735 \\
\hline$R_{\text {int }}$ & 0.0320 & 0.0779 \\
\hline Parameters & 365 & 327 \\
\hline Restraints & 212 & 50 \\
\hline Largest Peak & 1.899 & 4.326 \\
\hline Deepest Hole & -0.694 & -2.100 \\
\hline GooF & 1.036 & 1.092 \\
\hline$w R_{2}$ (all data) & 0.0819 & 0.1694 \\
\hline$w R_{2}$ & 0.0811 & 0.1571 \\
\hline$R_{l}$ (all data) & 0.0320 & 0.0671 \\
\hline$R_{1}$ & 0.0311 & 0.0576 \\
\hline
\end{tabular}




\section{Photophysical measurements.}

UV-Vis absorption spectra were recorded with a Varian Cary 300 double beam spectrometer and luminescence spectra were measured with a Horiba Jobin Yvon Fluorolog 3 steady-state fluorescence spectrometer. Photoluminescence quantum yields were determined with a Hamamatsu C9920-02 system. For decay time measurements a PicoQuant LDH-P-C-375 pulsed diode laser $\left(\lambda_{\mathrm{exc}}=372 \mathrm{~nm}\right.$, pulse width $\left.100 \mathrm{ps}\right)$ was applied as the excitation source. The emission signal was detected with a cooled photomultiplier attached to a FAST ComTec multichannel scalar card with a time resolution of $250 \mathrm{ps}$.

For sub-nanosecond decay time measurements, emission was excited with pulsed laser irradiation (pulse duration of about $80 \mathrm{fs}$, excitation wavelength $370 \mathrm{~nm}$ ) generated using a Ti-sapphire pumping laser (Libra Coherent) and a TOPAS-C (Light Conversion) optical parametric amplifier. The emission was detected with a Bruker 200is spectrograph connected to a Hamamatsu C7700 streak camera. The streak images were recorded on a Hamamatsu (ORCA-CR) CCD camera. For each sample a total of 8000 excitation shots were applied. The decay times were obtained from a global analysis of the thus collected time-resolved data. 
Table S2. Photophysical data for the free ligands HL1 - HL4.

\begin{tabular}{|c|c|c|c|c|}
\hline & Absorption $^{\text {a }}$ & Emission $^{b}$ & & \\
\hline & $\lambda \max / \mathbf{n m}\left(\varepsilon / \mathbf{M}^{-1} \cdot \mathbf{c m}^{-1}\right)$ & $\lambda_{\mathrm{em}}^{\mathrm{c}} / \mathrm{nm}$ & $\tau / \mathbf{n s}$ & $\phi P L \cdot 10^{2} / \%$ \\
\hline HL1 & 267 (7950), $339(22000)$ & 394 & $<3$ & 31 \\
\hline HL2 & 261 (7950), 327 (19050) & 390 & $<3$ & 33 \\
\hline HL3 & 263 (9350), 377 (25250) & 444 & $<3$ & 15 \\
\hline HL4 & 258 (9550), 373 (22800) & 441 & $<3$ & 13 \\
\hline
\end{tabular}

(a) Measured in $\mathrm{CHCl}_{3}$ at ambient temperature. (b) Measured in poly(methyl methacrylate) PMMA at ambient temperature. (c) Position of the highest-energy maximum in the observed vibronic progression. $\lambda_{\text {em }}$ corresponds roughly to the energy of the emitting state ("purely electronic" $0-0$ line).
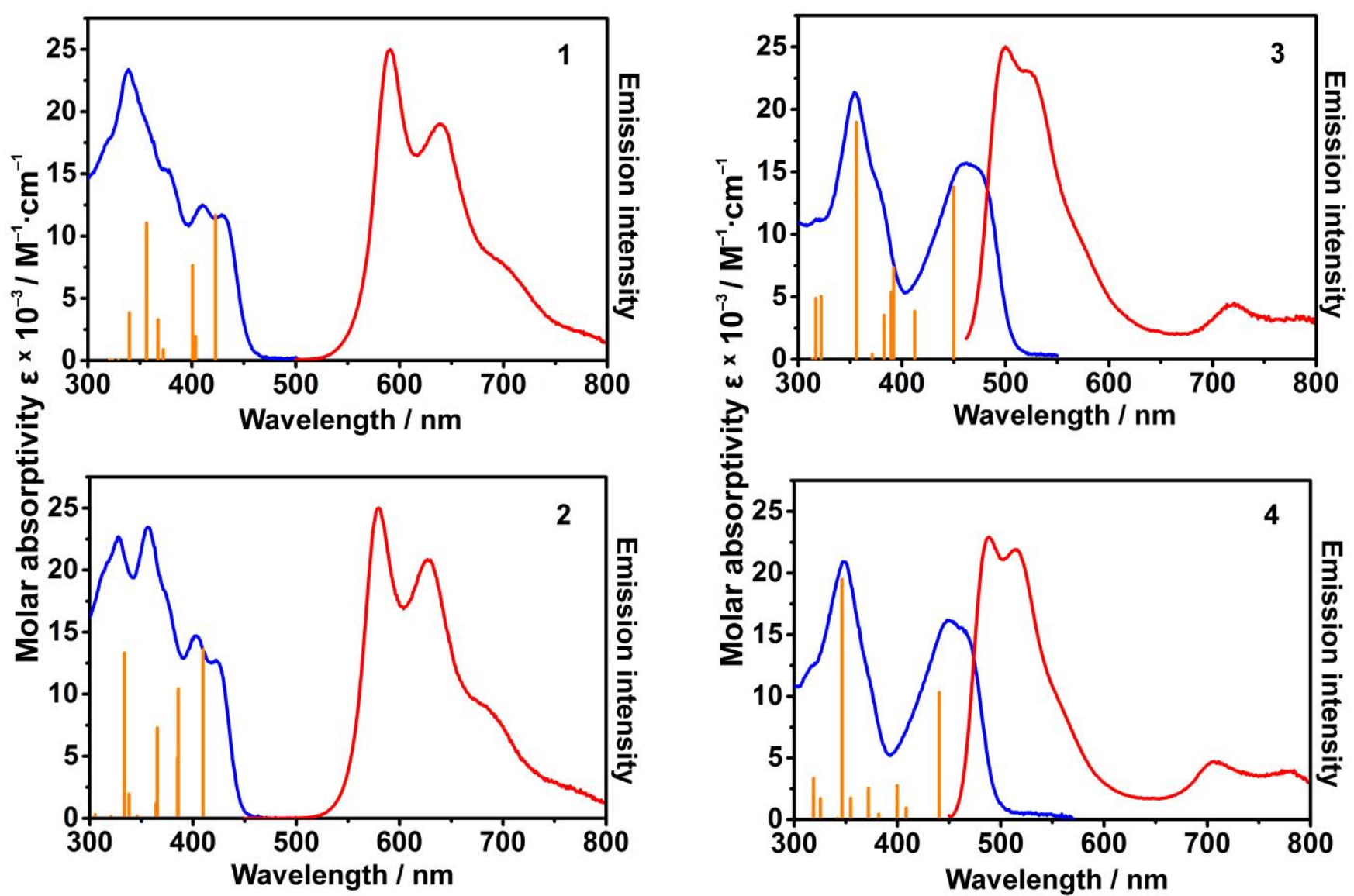

Figure S2. Ambient temperature UV-Vis absorption (blue lines) and luminescence spectra (red lines) of $\mathrm{Pt}$ complexes $1-4$. Absorption and emission spectra were recorded in $\mathrm{CHCl}_{3}$ solution and in poly(methyl methacrylate) (PMMA) films, respectively, at ambient temperature. Orange vertical bars represent vertical $\mathrm{S}_{\mathrm{n}} \leftarrow \mathrm{S}_{0}$ excitations as calculated at the TD-B3LYP/def2-TZVP theory level for the optimized ground state geometries. The bar sizes are proportional to the calculated oscillator strengths. 


\section{Computational details}

For all calculations, the Orca 3.0.1 package of programs was used ${ }^{4}$. The core electrons of Pt were described by the Stuttgart Dresden ${ }^{5}$ effective core potentials. (treating the valence electrons explicitly). The ground state geometries of the complexes were fully optimized without geometry constraints at the DFT/B3LYP ${ }^{6}$ level of theory using def2-TZVP basis set with polarization functions on all atoms. To investigate the vertical excitation energies of the low-lying excited states of the complexes, TD-DFT calculations were performed with the same basis sets and DFT functional as for geometry optimization. The $\mathrm{COSMO}^{7}$ solvation model was used to calculate the solvent effects in chloroform (dielectric constant $\varepsilon=4.9$, refractive index $\mathrm{nD}=1.45$ ). To accelerate TD-DFT calculations we employed the RIJCOSX ${ }^{8}$ approximation combining the RI-J method and the COSX approximation.

Table S3. XYZ coordinates of the optimized geometries of the platinum complexes $1-4$.

\section{Complex 1}

$\begin{array}{lc}\text { C } & -1.34964441402156 \\ \text { C } & -1.51507535582413 \\ \text { C } & -0.38647566599389 \\ \text { C } & 0.90251335411959 \\ \text { C } & 1.02244672496940 \\ \text { C } & -0.09581277388869 \\ \text { F } & -2.43956524724917 \\ \text { C } & 2.10377406772479 \\ \text { C } & 2.11859808586453 \\ \text { N } & 3.20250833765165 \\ \text { C } & 4.37964498610661 \\ \text { C } & 4.41036089382320 \\ \text { C } & 3.28807198332114 \\ \text { C } & 5.49241758091776 \\ \text { C } & 5.17942925216118 \\ \text { C } & 6.19240641848245 \\ \text { C } & 7.50821144353253 \\ \text { C } & 7.79270453489342 \\ \text { C } & 6.79868509266097 \\ \text { C } & 8.55048041403374 \\ \text { C } & 8.58544883244634 \\ \text { C } & 9.77299575254359 \\ \text { C } & 10.65565975840806 \\ \text { S } & 10.00756955978757 \\ \text { C } & 11.98391710504553 \\ \text { C } & 11.89942287724770 \\ \text { Pt } & 3.26471150557223 \\ \text { O } & 1.15912671697173 \\ \text { C } & 0.59380980669095 \\ \text { C } & 1.24268539460559 \\ \text { C } & 2.61971001487866 \\ \text { O } & 3.53287314973224 \\ \text { C } & 3.14307179370042 \\ \text { C } & -0.91371831170192 \\ \text { H } & -2.51029695121734 \\ & \end{array}$
7.54498221643856
6.61775111473645
6.09080519483852
6.47550579426588
7.41059620506511
7.95132010380096
8.06895251068640
5.89497304164461
4.56918255584161
3.97995842907910
4.66146808466991
6.01207654189173
6.62254244487105
3.84354395211253
2.47525890146941
1.60143552240130
2.04384262280840
3.41134110927198
4.29841981968679
1.07938501843802
$-0.26452325212660$
$-0.90603460067876$
$-0.06858047493277$
1.53503636577859
$-0.41575447583323$
$-0.84802088568195$
2.03449310039330
1.74041963480403
0.62688316641559
$-0.60421700379679$
$-0.81612358348724$
0.07612527656554
$-2.22204054709345$
0.67334473535271
6.32417231222868

3.54476899815925

2.53024888726092

1.91506182514813

2.30512463575670

3.34160871681385

3.96453154400301

4.14537241101516

1.66469145951231

1.23997295271195

0.72298876347625

0.60298766278865

0.95588858074648

1.47840691699050

0.15961389120955

$-0.02016909496064$

$-0.38928810439056$

$-0.58450672478255$

$-0.42053379809207$

$-0.05095992069147$

$-0.93017343428058$

$-0.64617551108718$

$-1.07192489742103$

$-1.69839555169817$

$-1.76330098628616$

$-2.30305183627812$

$-3.77510389484860$

0.20175721134884

0.30035765121460

0.07936938164316

$-0.11831411904103$

$-0.23101357687249$

$-0.22932129947635$

$-0.39339074603375$

0.03084392375222

2.22386023946882 


$\begin{array}{cc}\mathbf{H} & -0.51444400356321 \\ \mathbf{H} & 2.00435315299854 \\ \mathbf{H} & -0.00500062730165 \\ \mathbf{H} & 1.25112978551218 \\ \mathbf{H} & 5.32478937220100 \\ \mathbf{H} & 3.32597645495602 \\ \mathbf{H} & 5.95065494751283 \\ \mathbf{H} & 8.80411996122360 \\ \mathbf{H} & 7.05118435837495 \\ \mathbf{H} & 7.79548211095587 \\ \mathbf{H} & 9.97903436279977 \\ \mathbf{H} & 12.41971366767427 \\ \mathbf{H} & 12.66966343585230 \\ \mathbf{H} & 11.51646118198496 \\ \mathbf{H} & 11.22891226933417 \\ \mathbf{H} & 12.88368454599846 \\ \mathbf{H} & 0.61123298638551 \\ \mathbf{H} & 2.35507004826105 \\ \mathbf{H} & 3.90154011084217 \\ \mathbf{H} & 3.63139957332432 \\ \mathbf{H} & -1.21845820021219 \\ \mathbf{H} & -1.30134715404129 \\ \mathbf{H} & -1.35581905907044\end{array}$

C $\quad-1.71739019498110$

C $\quad-1.03501328717109$

C $\quad 0.17204483743267$

C 0.70003612013223

C $\quad-0.01797345168147$

C $\quad-1.22966606296367$

F $\quad-2.89422216178520$

C $\quad 1.97908248866907$

C $\quad 2.17119682496882$

N 3.32211557507237

C $\quad 4.41723424458955$

C $\quad 5.59486892336241$

C 5.40619128207639

C $\quad 6.46050715182563$

C $\quad 7.69953632085479$

C $\quad 7.87737096080814$

C $\quad 6.840766885553642$

C 8.74492097057141

C $\quad 8.57768918069614$

C $\quad 9.79605509997289$

C $\quad 10.90597176895675$

S 10.43773640531199

C 12.33938670157331

C 12.82944394970519

Pt 3.58044750509304

O 1.53155132719349
5.38242077111136

7.70447079821965

8.66541621715663

3.93026871475566

6.57368010751117

7.67176392787135

0.55977247160067

3.77195017067143

5.34374635998478

$-0.76759850061235$

$-1.95588908259891$

$-1.22761928065113$

0.43040011125911

$-0.04010271762560$

$-1.70088271667792$

$-1.13726849962988$

$-1.47547464563387$

$-2.96766470688749$

$-2.41012778227330$

$-2.32582869654300$

1.39603023955106

1.03054956002904

$-0.29353377251457$

Complex 2

6.66954941285303

5.56255827268995

5.22585391466123

5.97332161532778

7.09203822939043

7.44293349850509

6.99983042565516

5.54653692499395

4.19548315112381

3.68554605912764

4.47965176554944

3.71816317652928

2.31101643462534

1.49018303358798

2.01374812243646

3.40556014619954

4.24361147908636

1.10159043285446

$-0.07296482704620$

$-0.71973946876191$

$-0.05618124859575$

1.39366376701530

$-0.48808685106000$

$-1.21397772701758$

1.70070849908396

1.20008021732797
1.10706275001162

3.69096226359059

4.77189728114523

1.32806688600541

0.82603114226926

1.74206558469882

$-0.55172194583440$

$-0.55951251731562$

0.08490464471901

$-0.10764155274470$

$-0.90797435503942$

$-1.71458204501159$

$-2.20627607403829$

$-4.40137228110513$

$-3.89157073541751$

$-4.15078374480155$

$-0.21472195771495$

$-0.30515095610451$

0.36864931408552

$-1.36533418408141$

$-0.72871796947935$

0.98764373625505

$-0.20275544087164$

2.78583826224256

3.25753371410448

2.66060313168125

1.59857687615582

1.16093846590864

1.74640185304583

3.35951057287620

0.98248093416333

0.71025180609096

0.27097712204445

0.04599129469176

$-0.35514319105071$

$-0.36370435943464$

$-0.73631513809553$

$-1.12562784156227$

$-1.10035606298304$

$-0.72629416127612$

$-1.58922520094734$

$-2.27886091031770$

$-2.60081182376841$

$-2.15489456146658$

$-1.32762039223165$

$-2.24564947327174$

$-0.98259601060917$

0.07442957441816

0.38942113374379 


$\begin{array}{ll}\text { C } & 1.06415449469452 \\ \mathbf{C} & 1.81991333177066 \\ \mathbf{C} & 3.21106467576836 \\ \mathbf{O} & 4.03616392136725 \\ \mathbf{C} & 3.86376975107995 \\ \mathbf{C} & -0.44184456449600 \\ \mathbf{C} & 3.06457532920943 \\ \mathbf{C} & 4.27438241179313 \\ \mathbf{C} & 5.30672102234505 \\ \mathbf{C} & 4.66682326154918 \\ \mathbf{C} & 3.15314848498250 \\ \mathbf{H} & -1.44236447912332 \\ \mathbf{H} & 0.72427911967965 \\ \mathbf{H} & 0.35634394214155 \\ \mathbf{H} & -1.79614761700115 \\ \mathbf{H} & 1.37499684220211 \\ \mathbf{H} & 6.31982526287573 \\ \mathbf{H} & 8.82036584349139 \\ \mathbf{H} & 7.01057000154678 \\ \mathbf{H} & 7.60556027532903 \\ \mathbf{H} & 9.85196108374898 \\ \mathbf{H} & 12.98330145298459 \\ \mathbf{H} & 12.43923080841945 \\ \mathbf{H} & 12.20952865075926 \\ \mathbf{H} & 12.77996547163710 \\ \mathbf{H} & 13.86257128396479 \\ \mathbf{H} & 1.27248572547098 \\ \mathbf{H} & 4.40398813313455 \\ \mathbf{H} & 3.14132483389193 \\ \mathbf{H} & 4.59957392950718 \\ \mathbf{H} & -0.84075359635725 \\ \mathbf{H} & -0.82480753148529 \\ \mathbf{H} & -0.80128116305610 \\ \mathbf{H} & 5.50692170557585 \\ \mathbf{H} & 6.26167890928540 \\ \mathbf{H} & 4.89549553448735 \\ \mathbf{H} & 5.05707837693807 \\ \mathbf{H} & 2.63755585598381 \\ \mathbf{H} & 2.67298985808330\end{array}$

C $\quad 5.17795930696489$

C $\quad 5.17479066864137$

C $\quad 6.42338109935013$

C 7.35314098008511

S $\quad 6.68036236209078$

C $\quad-1.82971421203364$

C $\quad-1.96237059552472$

C $\quad-0.82019782201352$

C $\quad 0.44737393372658$

C $\quad 0.53552003942324$
0.02642382153869

$-1.15183851216527$

$-1.24076601622713$

$-0.27215614095073$

$-2.59975021491102$

$-0.06623524190968$

6.38608205140403

5.85715768713714

6.95342084654548

8.17441387341757

7.88394517902264

4.98742511428480

4.37396993189288

7.68504890458560

8.29763036220219

3.47700775422044

0.41769513582792

3.83394795468106

5.30893674990142

$-0.44126865839283$

$-1.65041296806104$

0.37231138655231

$-1.15361131925840$

$-2.08802108174733$

$-0.55848802083411$

$-1.54883333273703$

$-2.08310349228765$

$-2.73475988017456$

$-3.40519390459814$

$-2.65555634351838$

0.57863836302110

0.31293322179081

$-1.08214226350929$

7.15499762353217

6.68829499142453

9.11033503618517

8.25706963900411

8.36513447499328

8.24863783197446

\section{Complex 3}

3.61410933477284

2.22864695459610

1.69161868405577

2.64455006111204

4.25252000363249

6.92036867620277

5.87108071153396

5.35087380806855

5.87370613762570

6.92882709279238
0.28749101635126

0.17231755004175

0.06293030409798

$-0.03685027107523$

0.04085759371008

0.29946138116874

0.70889955125071

0.25596800776746

0.10559393067076

0.80730040189568

0.85482211785277

4.07814865992146

3.03594938676210

0.33730211000852

1.40163486193203

0.84818545612055

$-0.71659818607199$

$-1.41632921296737$

$-0.75801410363689$

$-2.57466435959109$

$-3.15033350941272$

$-2.44855097972895$

$-3.10713529530159$

$-0.77540433649172$

$-0.11152623967882$

$-1.10187538626135$

0.16912694419075

$-0.89862226025077$

0.16051175867755

0.84557063906456

$-0.48555982805387$

1.24989278380270

0.14424226752620

$-0.95245305393388$

0.55950859331691

0.29792412696572

1.82348208837611

0.01693176672895

1.76401011010259

0.50649392440897

0.41958022749619

0.81133311907093

1.18565054245032

1.08711463365387

$-2.87383593932772$

$-1.98074674508524$

$-1.38273287136280$

$-1.66390712777506$

$-2.58177774664678$ 


\begin{tabular}{|c|c|c|c|}
\hline C & -0.59505253146197 & 7.45838758063941 & -3.19022797739669 \\
\hline $\mathbf{F}$ & -2.93308070017430 & 7.44066568258523 & -3.45390094457191 \\
\hline $\mathbf{C}$ & 1.66782008776336 & 5.35101046578508 & -10105215585265 \\
\hline $\mathbf{C}$ & 1.88532083148642 & 3.98482161379597 & -0.84862831432060 \\
\hline $\mathbf{N}$ & 3.00523020077573 & 3.48249126872778 & -0.3160512412325 \\
\hline $\mathbf{C}$ & 4.01585849150250 & 4.31925499560206 & 0.0823016538767 \\
\hline $\mathbf{C}$ & 3.83578857195588 & 5.70353949059688 & -0.0065559743629 \\
\hline $\mathbf{C}$ & 2.67631349290847 & 6.21165567082980 & -0.54875416426 \\
\hline $\mathbf{P t}$ & 3.42568564939171 & 1.49648789788088 & -0.1561326980275 \\
\hline $\mathbf{O}$ & 1.44876853472847 & 0.91283135527226 & -0.5997479612177 \\
\hline C & 1.05176264575167 & -0.29248229233205 & \\
\hline $\mathbf{C}$ & 1.87104594875947 & -1.42529277229523 & -0.4416716445 \\
\hline C & 3.25617645895129 & -1.44381062486826 & -0.247307894665 \\
\hline $\mathbf{O}$ & 4.01796353571858 & -0.43769433952705 & -0.053750223560 \\
\hline C & 3.98099435337636 & 157816292 & -0.2443024 \\
\hline $\mathbf{C}$ & -0.44135933711311 & -0.47393172716728 & -0.6681725338630 \\
\hline $\mathbf{C}$ & 10.94566042453861 & 1.53917391250713 & 2.3485077685405 \\
\hline $\mathbf{C}$ & 10.957696 & 2.89 & 2.1 \\
\hline $\mathrm{C}$ & 9.71358775 & 3.418 & \\
\hline $\mathbf{C}$ & 8.72616283308735 & 2.46670617268162 & 1.6020000973622 \\
\hline $\mathbf{S}$ & 9.36895886455998 & 0.90244 & 2.0 \\
\hline $\mathbf{C}$ & 12.08134574 & 0.6 & \\
\hline $\mathbf{C}$ & 12.88158072886554 & 0.08 & \\
\hline $\mathbf{H}$ & 6.64764473848982 & $\$ 7901734$ & 0.7991715557283 \\
\hline $\mathbf{H}$ & -2.94459128240474 & 5.47918409380913 & -1.752058811955 \\
\hline $\mathbf{H}$ & -0.92088799151272 & 4.544 & -0.666 \\
\hline $\mathbf{H}$ & 1.50661614006057 & 7.33026598697604 & -2.843742497981 \\
\hline $\mathbf{H}$ & -0.52892229584626 & 8.26862144020290 & \\
\hline $\mathbf{H}$ & 1.15840511945420 & 3.25 & -1.1 \\
\hline $\mathbf{H}$ & 4.62016583727497 & 6.36272792417300 & 0.3405261974226 \\
\hline $\mathbf{H}$ & 2.53673842721131 & 7.28355536674307 & -0.6107674263008 \\
\hline $\mathbf{H}$ & 1.38144017105323 & -2.38596925149662 & \\
\hline $\mathbf{H}$ & 4.484 & -2.91 & 0.71405805 \\
\hline $\mathbf{H}$ & 4.75538111054563 & -2.74851242548148 & -1.014037187884 \\
\hline $\mathbf{H}$ & 3.31061742103722 & -3.60265011498864 & -0.431863875848 \\
\hline $\mathbf{H}$ & -0.92540233276276 & 352936654 & 0.10 \\
\hline $\mathbf{H}$ & -0.74538211631594 & -1.51388620599211 & -0.5608765228917 \\
\hline $\mathbf{H}$ & -0.79032307562603 & -0.09223121944165 & -1.6308449173953 \\
\hline $\mathbf{H}$ & 11.84194284116664 & 3.49871559616212 & 2.2943649014492 \\
\hline $\mathbf{H}$ & 9.54978858095072 & 4.46348619853333 & $1.500799906287 \mathrm{C}$ \\
\hline $\mathbf{H}$ & 11.70397742512851 & -0.17840524837760 & 3.3619666499372 \\
\hline $\mathbf{H}$ & 12.75063241524544 & 1.22387512892576 & 3.3979156177110 \\
\hline $\mathbf{H}$ & 13.32436933224050 & 0.88269158049091 & 0.9768549693857 \\
\hline $\mathbf{H}$ & 12.24276158352765 & -0.51017040464829 & 0.9110759772823 \\
\hline $\mathbf{H}$ & 13.68749082418638 & -0.56302615277262 & 1.929700267303 \\
\hline
\end{tabular}

\section{Complex 4}




\begin{tabular}{|c|c|c|c|}
\hline C & 5.17654281583967 & 3.10454057269790 & 0.24799240048164 \\
\hline $\mathbf{C}$ & 5.09105580261907 & 1.71678789895755 & 0.24224758471837 \\
\hline $\mathbf{C}$ & 6.30444551710007 & 1.11945651808510 & 0.64772895152958 \\
\hline $\mathbf{C}$ & 7.29564646960481 & 2.02587978919256 & 0.97025509019886 \\
\hline $\mathbf{S}$ & 6.73726731522672 & 3.66388409829562 & 0.76966704131919 \\
\hline C & -2.47017906214967 & 6.15380815365476 & -1.7597133156043 \\
\hline C & -2.29366290879794 & 5.20839415774075 & -0.7657298309402 \\
\hline $\mathbf{C}$ & -1.00046802614060 & 4.82364048365164 & -0.4346589141973 \\
\hline $\mathbf{C}$ & 0.11306175861384 & 5.36865679615555 & -1.0879525450415 \\
\hline $\mathbf{C}$ & -0.11517883654795 & 6.31825755528566 & -2.0918360074003 \\
\hline C & -1.40029818569719 & 6.71928411142997 & -2.432029121292 \\
\hline $\mathbf{F}$ & -3.72409826258645 & 6.53418163918449 & -2.086748716584 \\
\hline $\mathbf{C}$ & 1.48169380955480 & 4.91099192136954 & -0.744295750853 \\
\hline $\mathbf{C}$ & 1.73076531581089 & 3.54423218412513 & -0.6283594707746 \\
\hline $\mathbf{N}$ & 2.92202860559405 & 3.02981476276032 & -0.315766895828 \\
\hline $\mathbf{C}$ & 4.00021591586733 & 3.84882606609883 & -0.0807978541471 \\
\hline $\mathbf{P t}$ & 3.29174690043100 & 1.03478498076246 & -0.206701653394 \\
\hline $\mathbf{O}$ & 1.28043171259149 & 444197252 & \\
\hline $\mathbf{C}$ & 0.85823897029071 & 91667093 & \\
\hline C & 1.65568829301244 & -1.85553480839175 & -0.460864274373 \\
\hline $\mathbf{C}$ & 3.04656329626992 & -1.90324923723154 & -0.318771720134 \\
\hline $\mathbf{O}$ & 3.837601579 & 2019482 & \\
\hline $\mathbf{C}$ & 3.74251600813492 & -3.24023830307030 & -0.346946450862 \\
\hline $\mathbf{C}$ & -0.64023758796880 & -0.85127748987458 & -0.622877298409 \\
\hline $\mathbf{C}$ & 10.70948182326005 & 0.70952496167204 & 2.4041754362036 \\
\hline $\mathbf{C}$ & 10.86367862368080 & 17508488 & 2.0946777022024 \\
\hline $\mathbf{C}$ & 9.69970013255343 & 2.63253798932022 & 1.5555065281511 \\
\hline C & 8.63567620458032 & 1.76799782584032 & 1.4464404164389 \\
\hline $\mathbf{S}$ & 9.09946551167078 & 0.19321466047258 & 2.025 \\
\hline $\mathbf{C}$ & 2.57619354929867 & 22086778 & -0.51 \\
\hline $\mathbf{C}$ & 3.81957571445458 & 5.23099852967889 & -0.189546483705 \\
\hline $\mathbf{C}$ & 4.83550538128201 & 6.32003098951107 & 0.0490166332334 \\
\hline $\mathbf{C}$ & 4.14306785358921 & 32801554 & \\
\hline C & 2.63208889861431 & 7.27083916986479 & \\
\hline C & 11.72658373950999 & -0.25028516992773 & 2.9433624455858 \\
\hline $\mathbf{C}$ & 12.35853616743630 & -1.14913338259942 & 1.86 \\
\hline $\mathbf{H}$ & 6.45061613874513 & 0.04946393571488 & 0.7044623561714 \\
\hline $\mathbf{H}$ & -3.15474527709328 & 4.79104671771251 & -0.261155012388 \\
\hline $\mathbf{H}$ & -0.85349930342663 & 4.09545524459701 & 0.3527869026151 \\
\hline $\mathbf{H}$ & 0.72023428948685 & 6.73069849758685 & -2.640763281603 \\
\hline $\mathbf{H}$ & -1.57839363921111 & 7.44604836214972 & -3.213303170423 \\
\hline $\mathbf{H}$ & 0.95081482492766 & 2.81707935387845 & $-0.809132904725^{\prime}$ \\
\hline $\mathbf{H}$ & 1.14407735271739 & -2.80461708833700 & -0.524569961563 \\
\hline $\mathbf{H}$ & 4.27524272911023 & -3.39865061148391 & 0.5929524099496 \\
\hline $\mathbf{H}$ & 4.48968115798581 & -3.23573166710862 & -1.143214073340 \\
\hline $\mathbf{H}$ & 3.04824351431783 & -4.06192224514053 & -0.512704017724 \\
\hline $\mathbf{H}$ & -1.09918443842973 & -0.29584979735292 & 0.1971639390395 \\
\hline $\mathbf{H}$ & -0.96267485656588 & -1.88994878455333 & -0.573619831913 \\
\hline $\mathbf{H}$ & -0.99922516770209 & -0.40534670056604 & -1.553260505082 \\
\hline $\mathbf{H}$ & 11.79290080145299 & 2.56509249129934 & 2.2517138965930 \\
\hline H & 9.64828356888476 & 3.66750651451028 & 1.2454751528751 \\
\hline
\end{tabular}




$\begin{array}{cccc}\mathbf{H} & 5.79064027858539 & 6.11884704766455 & -0.43944911861177 \\ \mathbf{H} & 5.04731920624737 & 6.41814876037205 & 1.11959670422541 \\ \mathbf{H} & 4.46497654515133 & 7.75165871552819 & -1.53941515836958 \\ \mathbf{H} & 4.39130958340544 & 8.48250481649148 & 0.05586045354284 \\ \mathbf{H} & 2.09974199644204 & 7.74966037134937 & -1.30806467311559 \\ \mathbf{H} & 2.16006895294043 & 7.62736932877118 & 0.43348546351064 \\ \mathbf{H} & 11.27168232009416 & -0.87738930503246 & 3.71573256714078 \\ \mathbf{H} & 12.50868282637242 & 0.33134575975598 & 3.43876529130816 \\ \mathbf{H} & 12.88350647990000 & -0.55029755685223 & 1.12258445242213 \\ \mathbf{H} & 11.59969532363617 & -1.73812158283943 & 1.35096975857267 \\ \mathbf{H} & 13.07406397585862 & -1.83950713190282 & 2.32192667570785\end{array}$



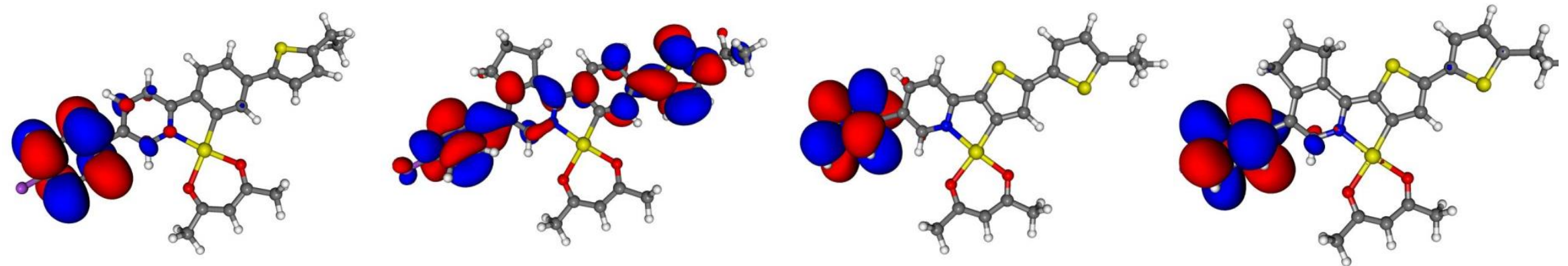

$-0.7807$

$-0.7164$

$-0.7622$

$-0.7237$

LUMO+3
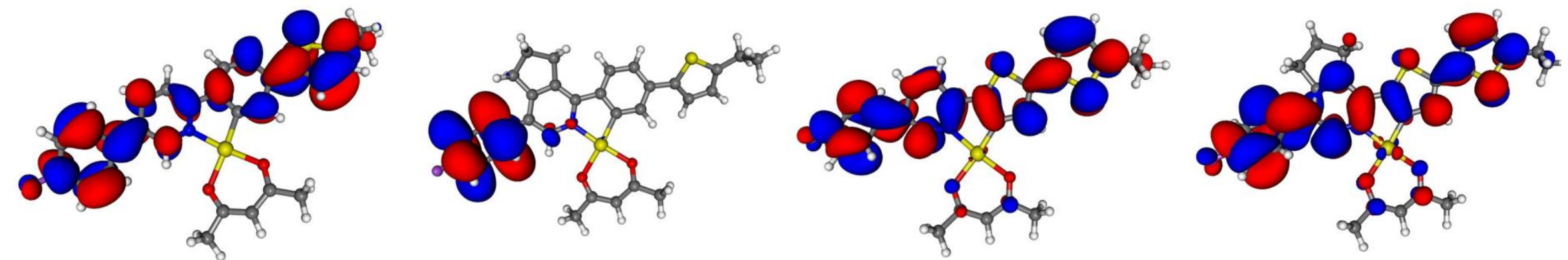

$-0.8220$

$-0.7525$

$-0.8529$

$-0.8056$
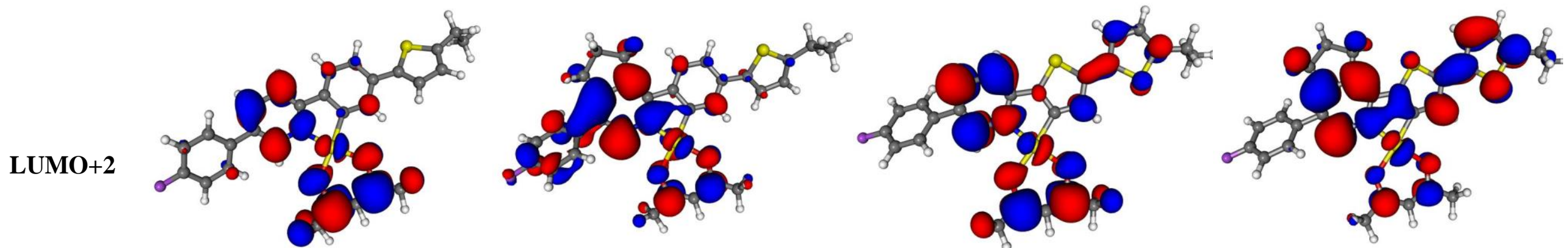

$-1.0401$

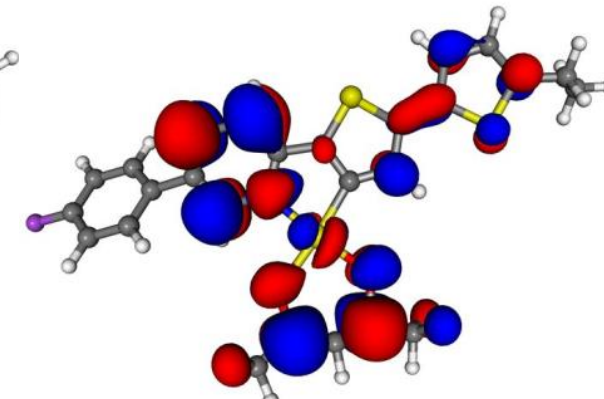




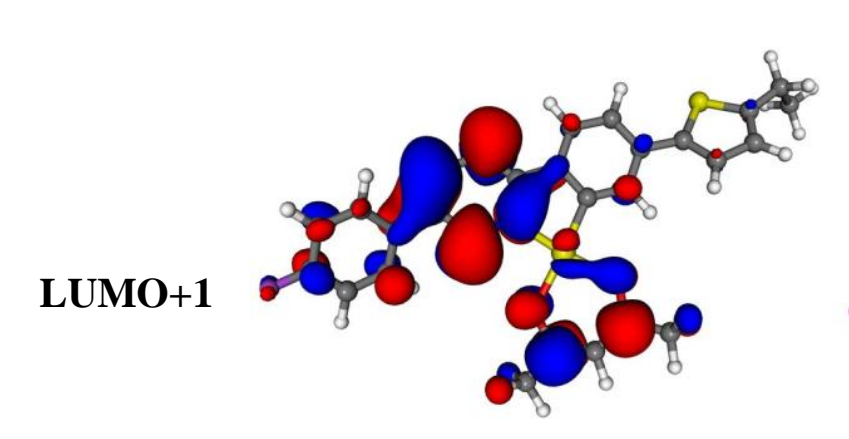

$-1.4118$

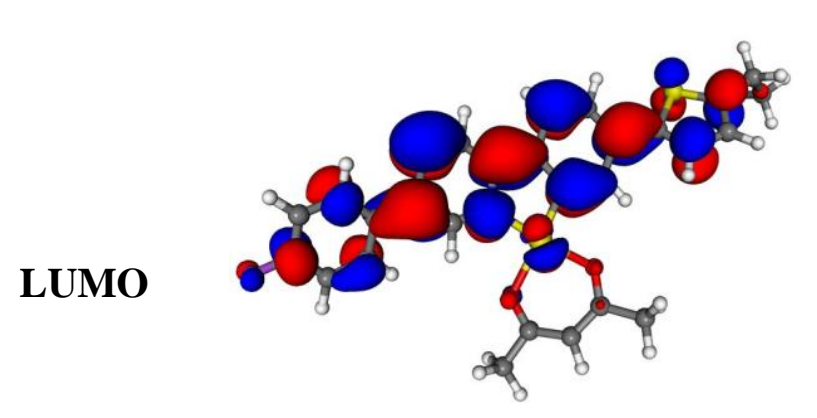

$-2.0739$

HOMO

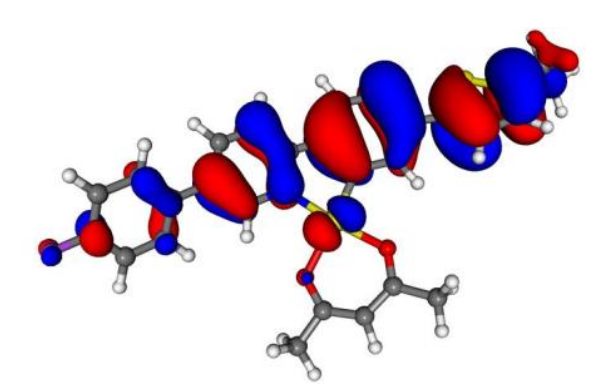

$-5.5094$

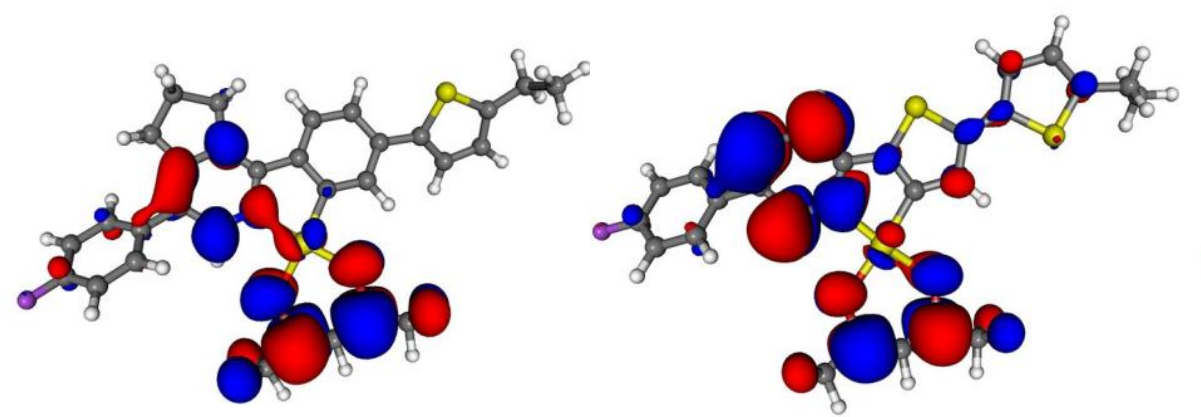

$-1.2757$

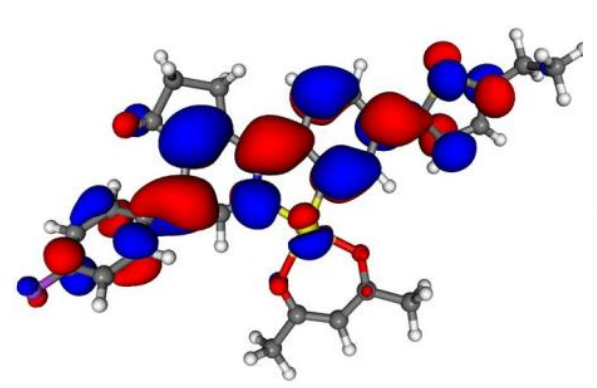

$-1.9353$

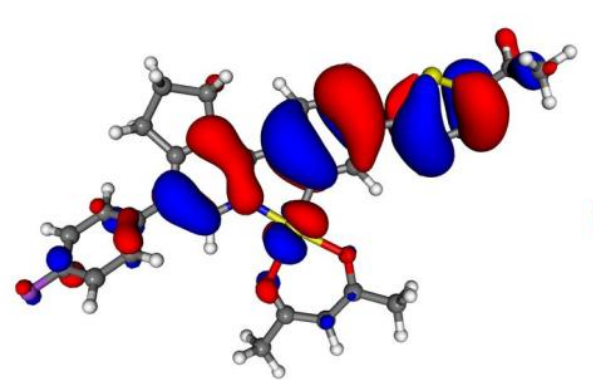

$-5.5100$
$-1.4076$

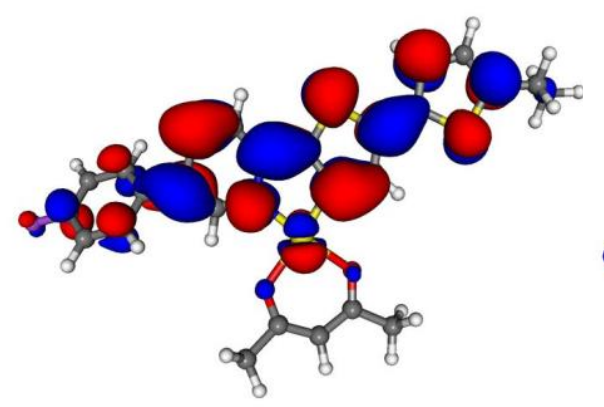

$-2.1222$

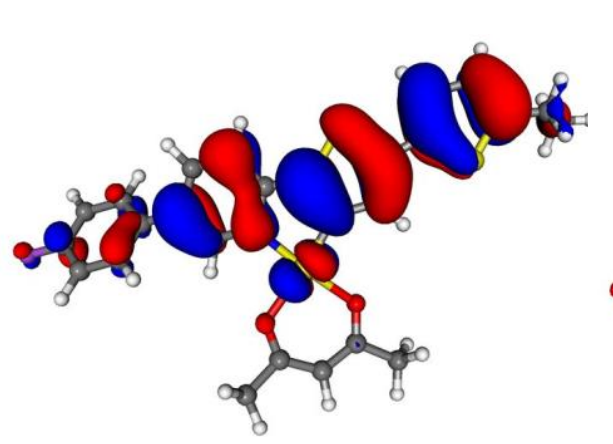

$-5.2108$

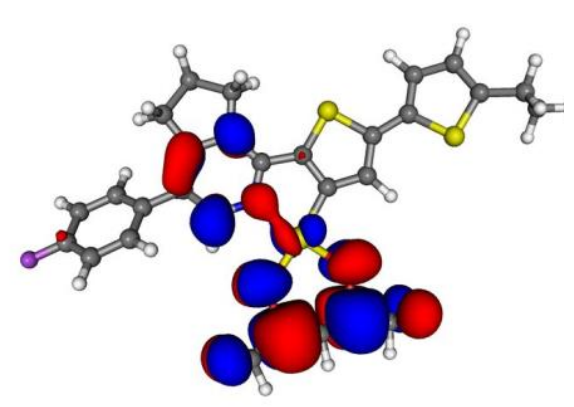

$-1.3034$

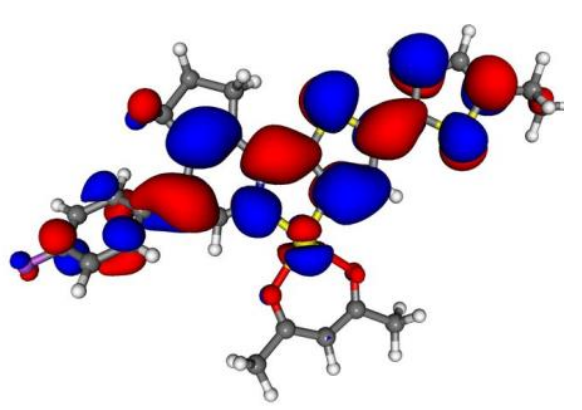

$-2.0278$

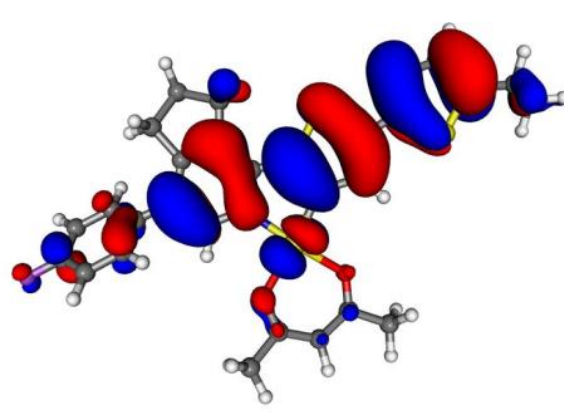

$-5.1517$ 


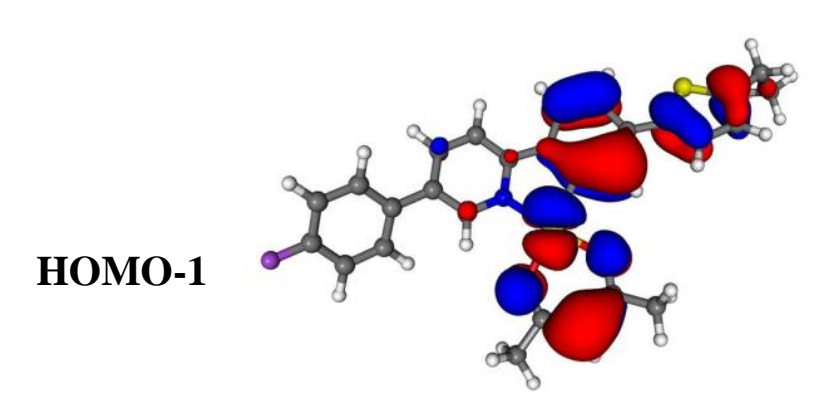

$-5.8763$

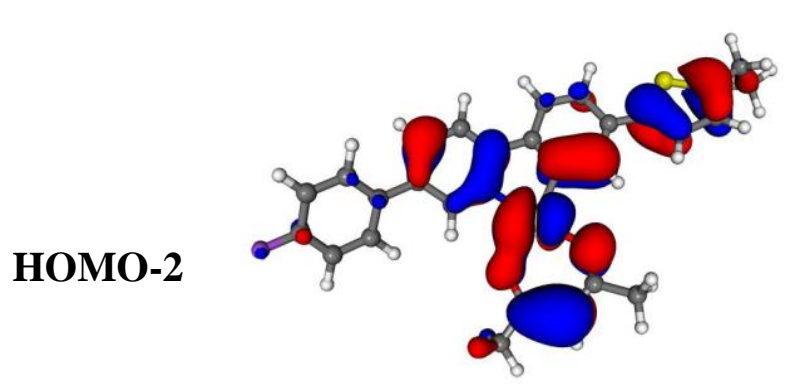

$-6.2156$

HOMO-3

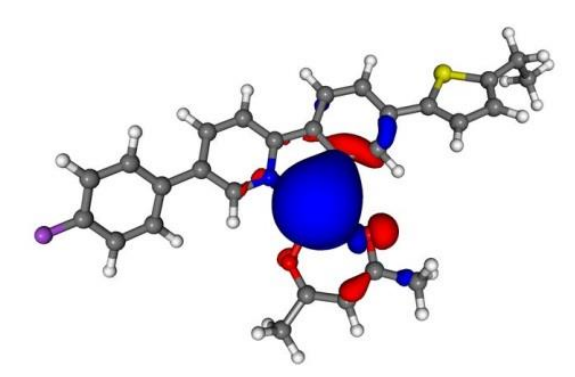

$-6.3688$

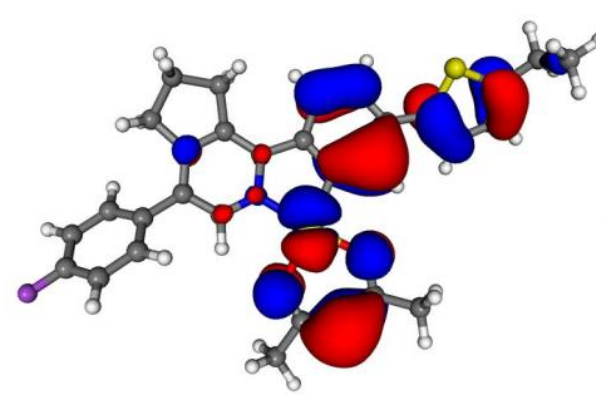

$-5.8197$

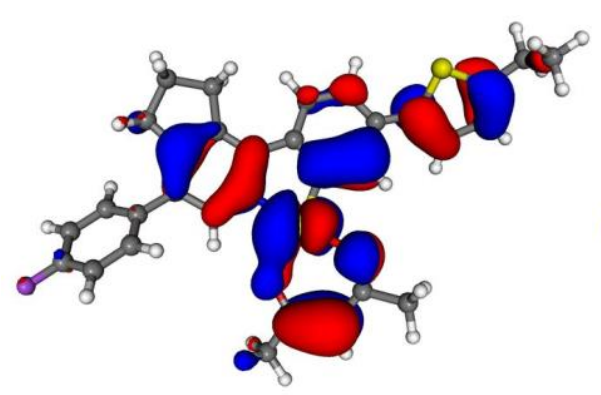

$-6.1375$

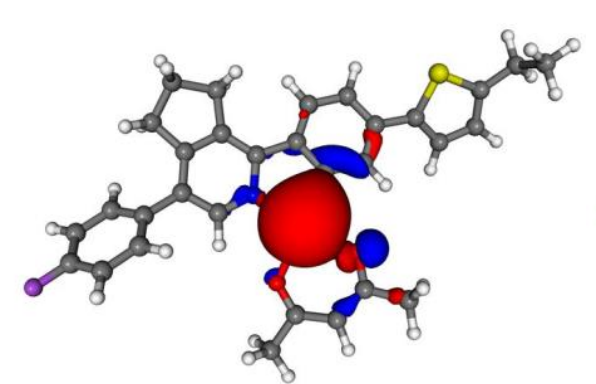

$-6.3070$

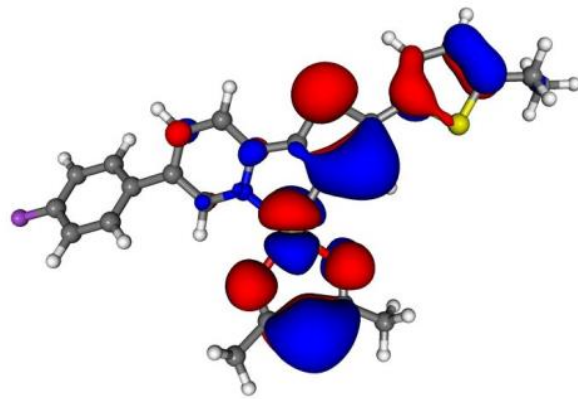

$-5.9986$

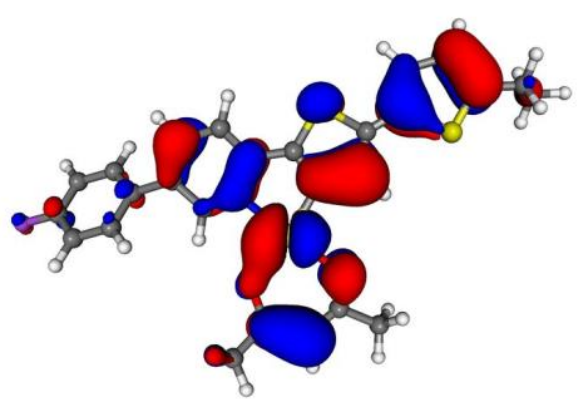

$-6.3376$

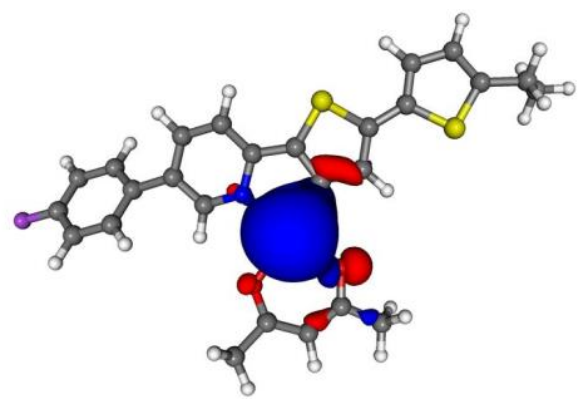

$-6.4383$

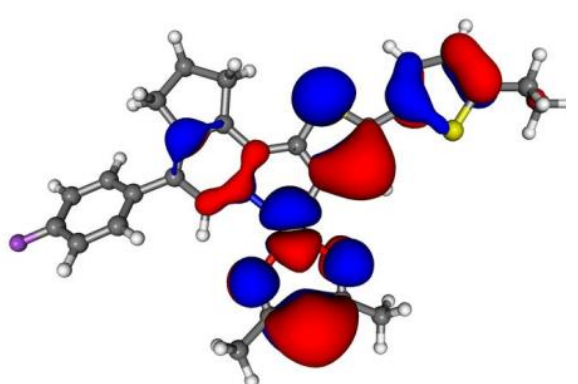

$-5.9340$

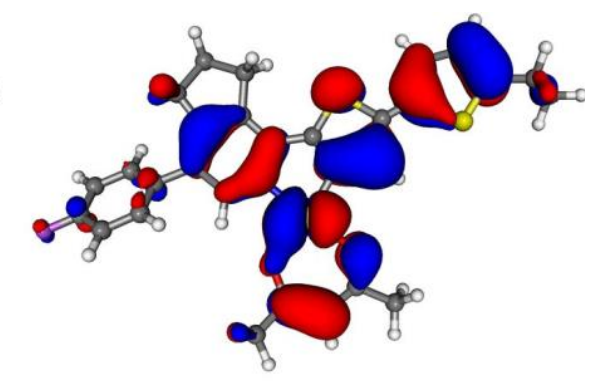

$-6.2553$

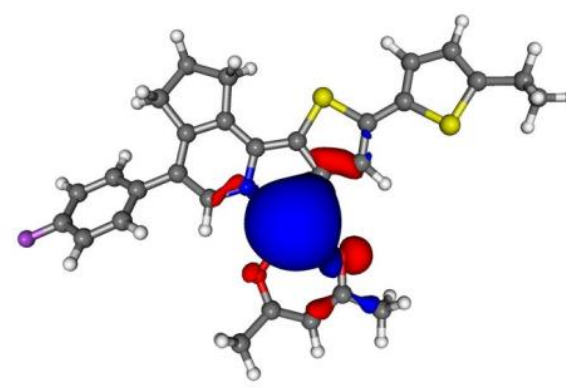

$-6.3764$ 


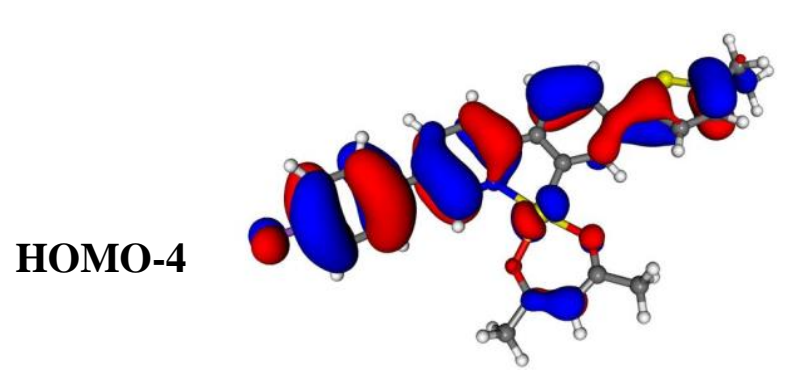

$-6.6302$

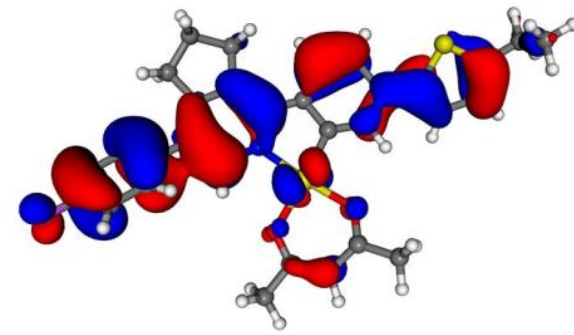

$-6.6065$

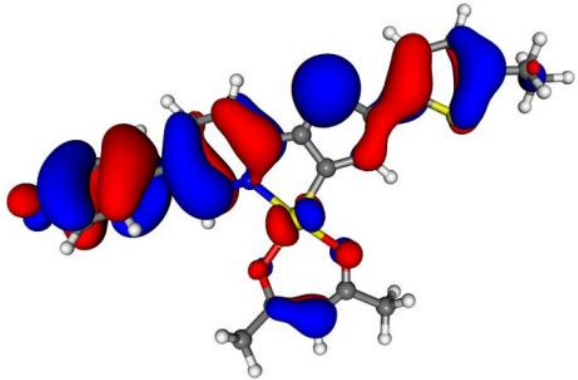

$-6.6425$

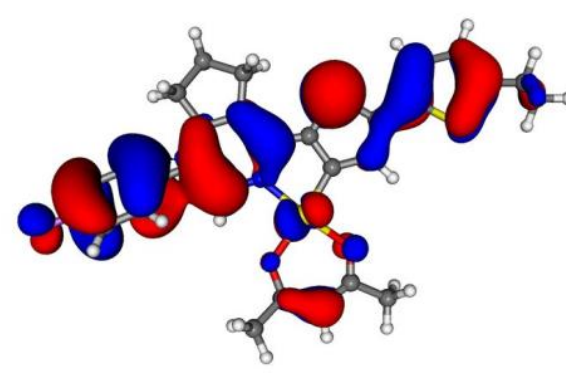

$-6.6148$

Figure S3. Molecular orbital contour plots resulting from DFT calculations (B3LYP/def2-TZVP) in the ground state geometries of complexes $\mathbf{1}-\mathbf{4}$. Orbital energies are given in $\mathrm{eV}$.

\section{Complex 1}

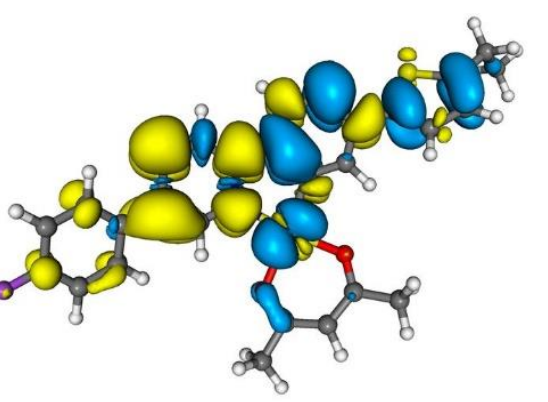

$\mathbf{S}_{2}$

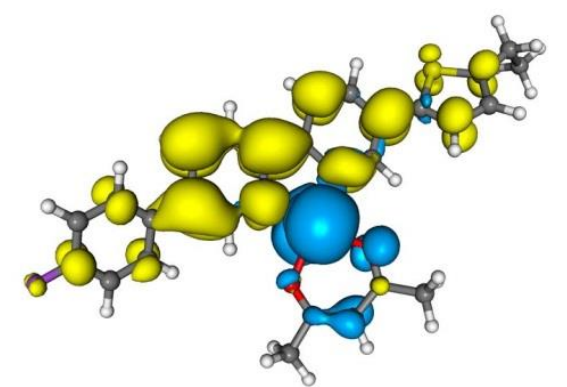

Complex 2
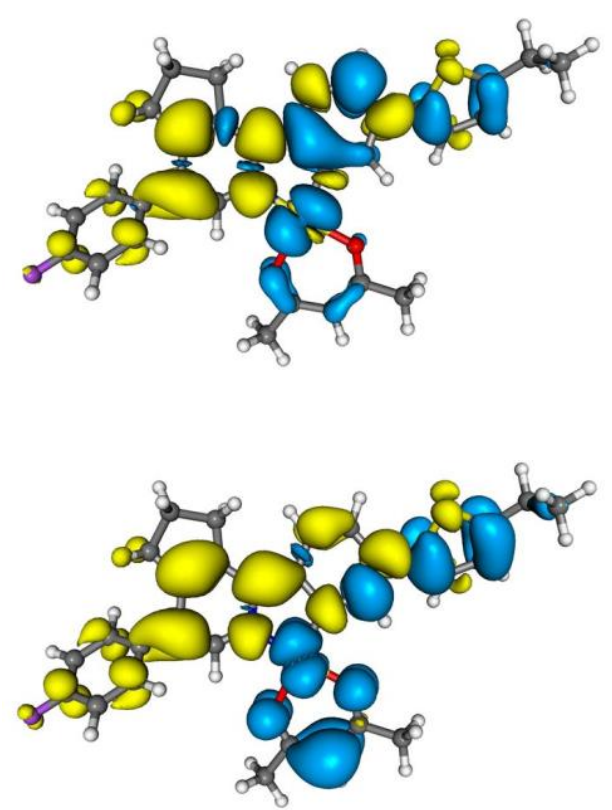

Complex 3
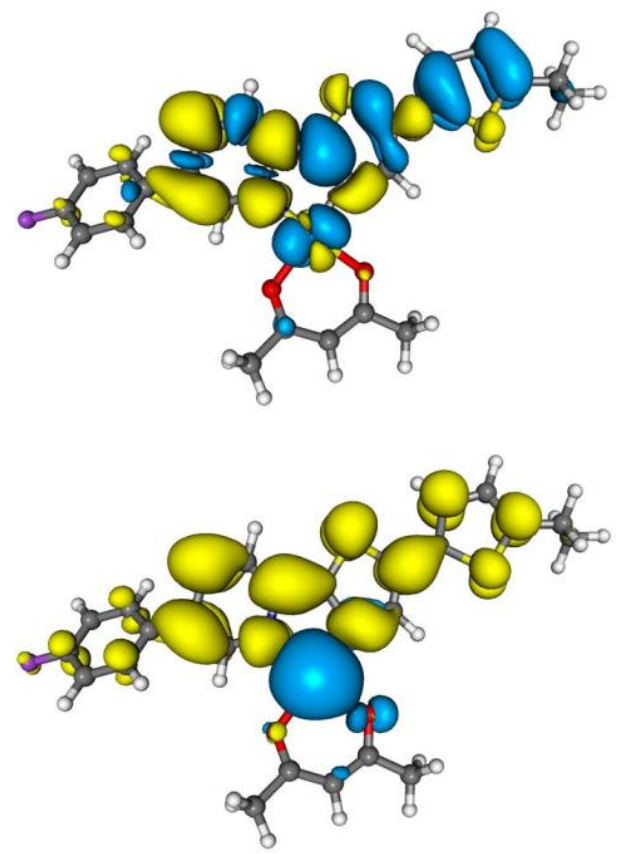

Complex 4
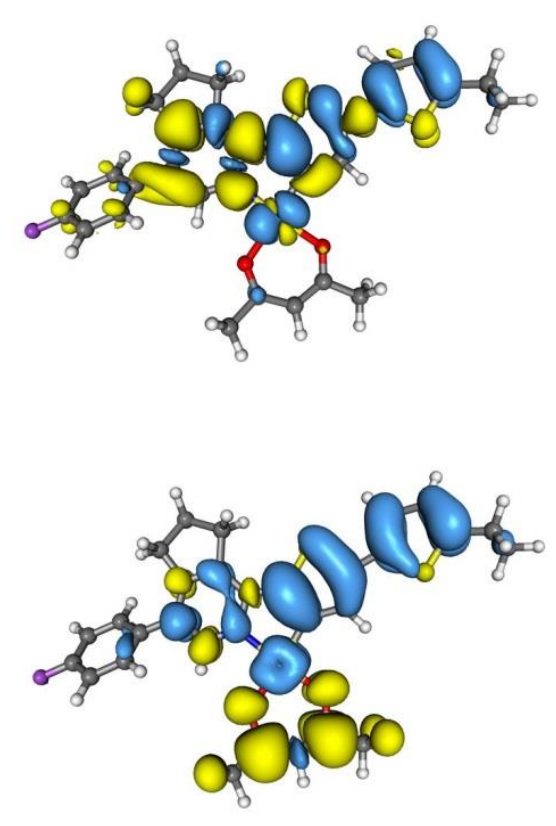


$$
\text { " od }
$$


-

20?

ros sos

1.

1. for

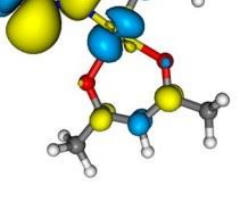

\%
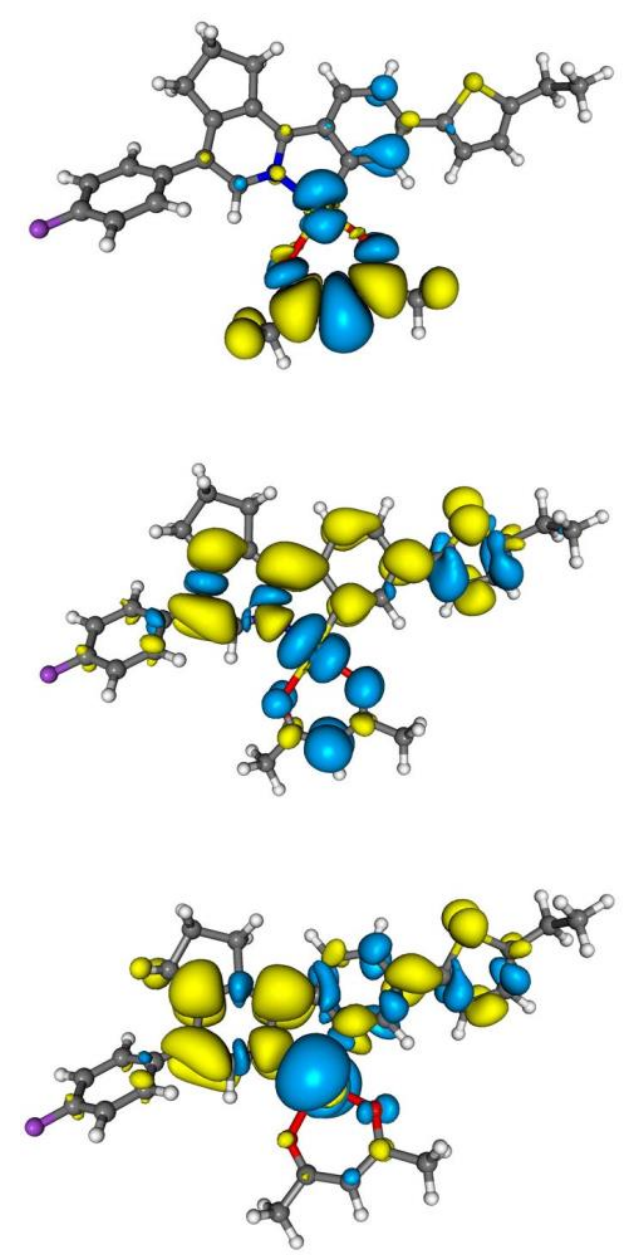
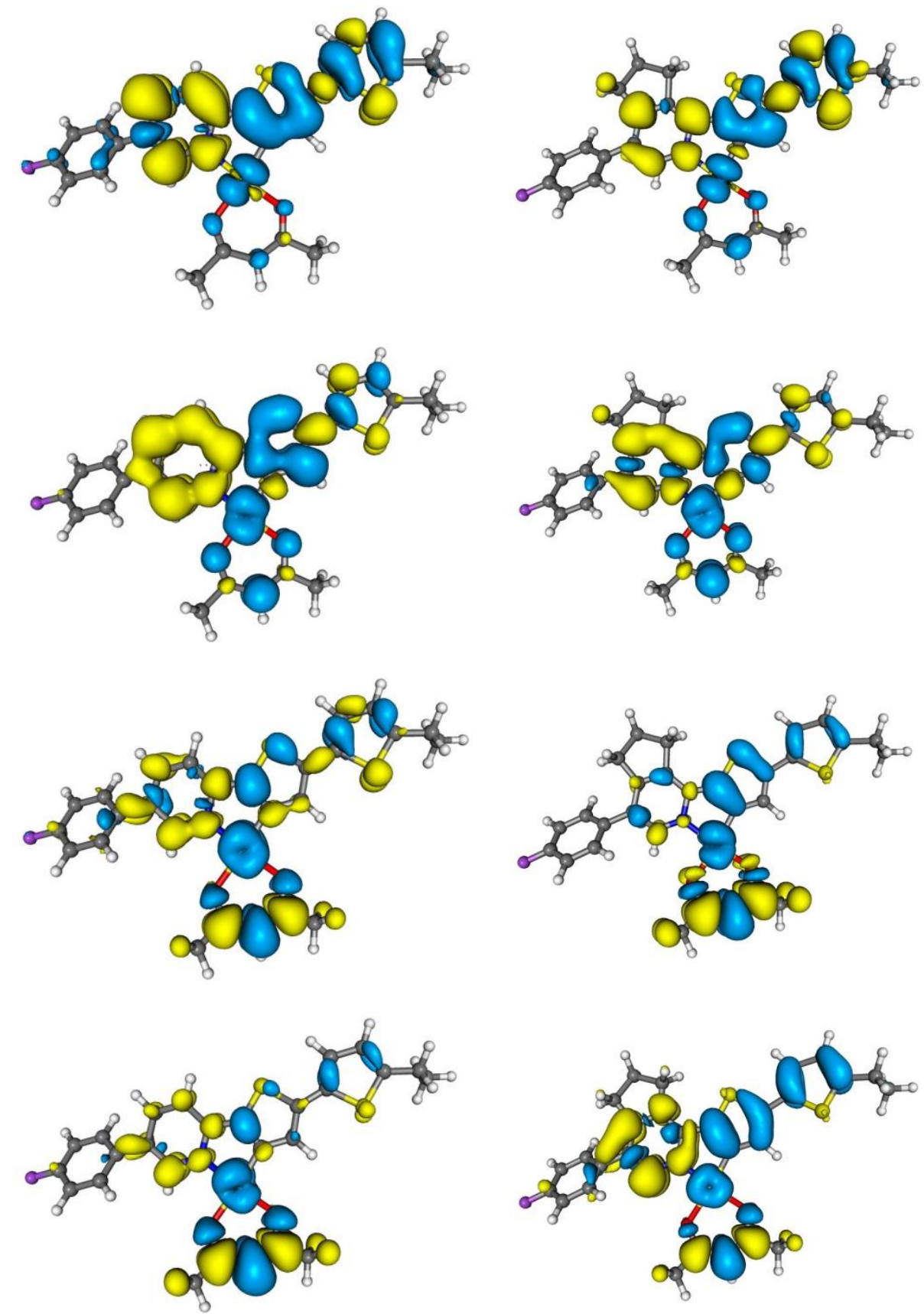
Figure S4. Contour plots of the excited state electron density differences of five lowest singlet $S_{n}$ and triplet $T_{n}$ states of Pt complexes $\mathbf{1}-\mathbf{4}$ resulting from TD-DFT caclulations (B3LYP/def2-TZVP). A local increase and decrease of the electron density in respect to the ground state $\mathrm{S}_{0}$ are marked with blue and yellow isosurface colors, respectively. 
Table S4. Total Pt contributions (\%) to MOs of the complexes 1 - 4 resulting from Mulliken MO analyses.

\begin{tabular}{|c|c|c|c|c|}
\hline \multirow{2}{*}{ Orbitals } & \multicolumn{4}{|c|}{ Heavy atom (Pt) contribution (\%) } \\
\cline { 2 - 5 } & Complex 1 & Complex 2 & Complex 3 & Complex 4 \\
\hline LUMO+4 & 0.2 & 0.3 & 0.1 & 0.2 \\
\hline LUMO+3 & 0.1 & 0.2 & 0.5 & 0.8 \\
\hline LUMO+2 & 3.1 & 3.2 & 3.3 & 3.0 \\
\hline LUMO+1 & 1.1 & 1.5 & 1.1 & 1.7 \\
\hline LUMO & 3.9 & 3.9 & 4.7 & 4.4 \\
\hline HOMO & 5.5 & 8.3 & 7.0 & 7.4 \\
\hline HOMO-1 & 31.9 & 29.8 & 29.7 & 30.5 \\
\hline HOMO-2 & 40.5 & 42.6 & 38.6 & 40.7 \\
\hline HOMO-3 & 91.2 & 91.3 & 92.7 & 92.5 \\
\hline HOMO-4 & 4.7 & 4.2 & 3.2 & 3.7 \\
\hline
\end{tabular}

Table S5. Pt atomic orbital contributions to the molecular orbitals resulting from Mulliken MO analyses.

\begin{tabular}{|c|c|c|c|c|c|c|c|c|}
\hline \multirow{2}{*}{ MO } & \multicolumn{2}{|c|}{ Complex 1} & \multicolumn{2}{|c|}{ Complex 2} & \multicolumn{2}{|c|}{ Complex 3} & \multicolumn{2}{|c|}{ Complex 4} \\
\hline & $\mathrm{AO}$ of $\mathrm{Pt}$ & Contr. & $\mathrm{AO}$ of $\mathrm{Pt}$ & Contr. & $\mathrm{AO}$ of $\mathrm{Pt}$ & Contr. & $\mathrm{AO}$ of $\mathrm{Pt}$ & Contr. \\
\hline \multirow{2}{*}{ 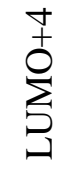 } & 5 py & 0.1 & $6 s$ & 0.1 & & 0.0 & $3 \mathrm{dx} 2 \mathrm{y} 2$ & 0.1 \\
\hline & SUM & 0.1 & SUM & 0.1 & SUM & 0.0 & SUM & 0.1 \\
\hline \multirow{5}{*}{ 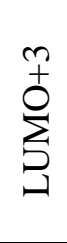 } & & 0.0 & $3 \mathrm{~d} x 2 \mathrm{y} 2$ & 0.1 & $5 \mathrm{pz}$ & 0.2 & $6 s$ & 0.2 \\
\hline & & & & & $3 \mathrm{dxz}$ & 0.1 & $3 \mathrm{dxz}$ & 0.1 \\
\hline & & & & & & & $3 \mathrm{dyz}$ & 0.1 \\
\hline & & & & & & & $3 \mathrm{dx} 2 \mathrm{y} 2$ & 0.2 \\
\hline & SUM & 0.0 & SUM & 0.1 & SUM & 0.3 & SUM & 0.6 \\
\hline \multirow{12}{*}{ 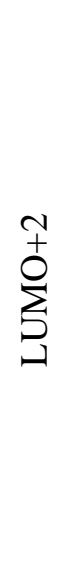 } & $4 \mathrm{pz}$ & 0.1 & $4 \mathrm{pz}$ & 0.1 & $4 \mathrm{pz}$ & 0.1 & $4 \mathrm{pz}$ & 0.2 \\
\hline & $5 \mathrm{pz}$ & 0.1 & $5 \mathrm{pz}$ & 0.4 & $5 \mathrm{pz}$ & 0.2 & $5 \mathrm{pz}$ & 0.6 \\
\hline & 5 py & 0.1 & $3 \mathrm{dxz}$ & 0.1 & $5 p x$ & 0.1 & $5 \mathrm{px}$ & 0.1 \\
\hline & $3 \mathrm{dz} 2$ & 0.3 & $3 \mathrm{dyz}$ & 1.6 & $3 \mathrm{dz} 2$ & 0.1 & $3 \mathrm{dyz}$ & 1.7 \\
\hline & $3 \mathrm{dxz}$ & 0.2 & $3 \mathrm{~d} x 2 \mathrm{y} 2$ & 0.2 & $3 \mathrm{dxz}$ & 0.6 & $3 \mathrm{dxy}$ & 0.1 \\
\hline & $3 \mathrm{dyz}$ & 1.6 & $3 \mathrm{dxy}$ & 0.4 & $3 \mathrm{dyz}$ & 1.6 & 4 dyz & 0.3 \\
\hline & $3 \mathrm{dxy}$ & 0.1 & $4 d y z$ & 0.3 & $3 d x y$ & 0.3 & $5 \mathrm{dyz}$ & -0.2 \\
\hline & $4 \mathrm{dz} 2$ & 0.1 & $5 \mathrm{dyz}$ & -0.1 & $4 \mathrm{dxz}$ & 0.1 & & \\
\hline & $4 \mathrm{dxz}$ & 0.1 & & & $4 \mathrm{dyz}$ & 0.3 & & \\
\hline & $4 d y z$ & 0.3 & & & $4 d x y$ & 0.1 & & \\
\hline & $5 \mathrm{dxz}$ & 0.1 & & & $5 \mathrm{dyz}$ & -0.1 & & \\
\hline & $5 \mathrm{dyz}$ & -0.1 & & & & & & \\
\hline
\end{tabular}




\begin{tabular}{|c|c|c|c|c|c|c|c|c|}
\hline & SUM & 3.0 & SUM & 3.0 & SUM & 3.4 & SUM & 2.8 \\
\hline \multirow{7}{*}{ 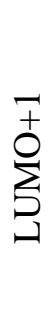 } & $4 \mathrm{pz}$ & 0.1 & $3 \mathrm{dz} 2$ & 0.1 & $4 \mathrm{pz}$ & 0.1 & $3 \mathrm{dz} 2$ & 0.1 \\
\hline & $5 \mathrm{pz}$ & 0.1 & $3 \mathrm{dxz}$ & 0.8 & $5 \mathrm{pz}$ & 0.1 & $3 \mathrm{dxz}$ & 1.0 \\
\hline & $3 \mathrm{dxz}$ & 0.5 & 3dyz & 0.1 & $3 \mathrm{dz} 2$ & 0.1 & 3dyz & 0.1 \\
\hline & $3 \mathrm{dyz}$ & 0.2 & $4 \mathrm{dxz}$ & 0.1 & $3 \mathrm{dxz}$ & 0.4 & $4 \mathrm{dxz}$ & 0.2 \\
\hline & $3 \mathrm{dx} 2 \mathrm{y} 2$ & 0.1 & $5 \mathrm{dxz}$ & 0.1 & $3 \mathrm{dyz}$ & 0.1 & & \\
\hline & $4 \mathrm{dxz}$ & 0.1 & & & $4 \mathrm{dxz}$ & 0.1 & & \\
\hline & SUM & 1.1 & SUM & 1.2 & SUM & 0.9 & SUM & 1.4 \\
\hline \multirow{11}{*}{ 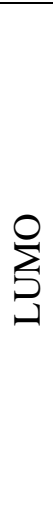 } & $4 \mathrm{pz}$ & 0.3 & $4 \mathrm{pz}$ & 0.3 & $4 \mathrm{pz}$ & 0.3 & $4 \mathrm{pz}$ & 0.3 \\
\hline & $5 \mathrm{pz}$ & 1.4 & $5 \mathrm{pz}$ & 1.5 & $5 \mathrm{pz}$ & 1.7 & $5 \mathrm{pz}$ & 1.8 \\
\hline & 5 py & 0.1 & $5 p x$ & 0.2 & $5 \mathrm{px}$ & 0.2 & $5 \mathrm{px}$ & 0.1 \\
\hline & $3 \mathrm{dz} 2$ & 0.1 & $3 \mathrm{dxz}$ & 0.4 & $3 \mathrm{dz} 2$ & 0.1 & $3 \mathrm{dz} 2$ & 0.1 \\
\hline & $3 \mathrm{dxz}$ & 0.6 & $3 \mathrm{dyz}$ & 1.1 & $3 \mathrm{dxz}$ & 0.4 & $3 \mathrm{dxz}$ & 0.5 \\
\hline & 3dyz & 0.8 & $4 \mathrm{dxz}$ & 0.1 & 3dyz & 1.1 & 3dyz & 1.1 \\
\hline & $3 \mathrm{dx} 2 \mathrm{y} 2$ & 0.1 & 4dyz & 0.3 & $3 d x y$ & 0.2 & $3 d x y$ & 0.1 \\
\hline & $4 \mathrm{dxz}$ & 0.1 & $5 \mathrm{dxz}$ & -0.1 & $4 \mathrm{dxz}$ & 0.1 & $4 \mathrm{dxz}$ & 0.1 \\
\hline & $4 \mathrm{dyz}$ & 0.2 & & & 4dyz & 0.3 & 4dyz & 0.3 \\
\hline & $5 \mathrm{dxz}$ & -0.1 & & & $5 \mathrm{dxz}$ & -0.1 & $5 \mathrm{dxz}$ & -0.1 \\
\hline & SUM & 3.6 & SUM & 3.8 & SUM & 4.3 & SUM & 4.3 \\
\hline \multirow{9}{*}{$\sum_{\substack{0 \\
0}}^{0}$} & $3 \mathrm{dz} 2$ & 0.6 & $3 \mathrm{dz} 2$ & 0.8 & $3 \mathrm{dz} 2$ & 1.3 & $3 \mathrm{dz} 2$ & 0.8 \\
\hline & $3 \mathrm{dxz}$ & 2.8 & $3 \mathrm{dxz}$ & 4.5 & $3 \mathrm{dxz}$ & 3.3 & $3 \mathrm{dxz}$ & 4.2 \\
\hline & 3dyz & 0.1 & $3 \mathrm{~d} x 2 \mathrm{y} 2$ & 0.5 & $3 \mathrm{~d} x 2 \mathrm{y} 2$ & 0.5 & $3 \mathrm{~d} x 2 \mathrm{y} 2$ & 0.4 \\
\hline & $3 \mathrm{dxy}$ & 0.4 & $3 \mathrm{dxy}$ & 0.1 & $4 \mathrm{dz2}$ & 0.5 & $4 \mathrm{dz} 2$ & 0.3 \\
\hline & $4 \mathrm{dz} 2$ & 0.2 & $4 \mathrm{dz} 2$ & 0.3 & $4 \mathrm{dxz}$ & 1.1 & $4 \mathrm{dxz}$ & 1.5 \\
\hline & $4 \mathrm{dxz}$ & 1.0 & $4 \mathrm{dxz}$ & 1.7 & $3 d x y$ & 0.2 & $4 \mathrm{dx} 2 \mathrm{y} 2$ & 0.1 \\
\hline & $4 \mathrm{dxy}$ & 0.1 & $4 \mathrm{dx} 2 \mathrm{y} 2$ & 0.2 & & & $5 \mathrm{dxz}$ & 0.1 \\
\hline & $5 \mathrm{dxz}$ & 0.1 & $5 \mathrm{dxz}$ & 0.2 & & & & \\
\hline & SUM & 5.3 & SUM & 8.3 & SUM & 6.9 & SUM & 7.4 \\
\hline \multirow{15}{*}{ 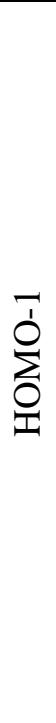 } & $5 \mathrm{pz}$ & -0.1 & $3 \mathrm{dz} 2$ & 1.3 & $3 \mathrm{dz} 2$ & 1.7 & $3 \mathrm{dz} 2$ & 1.1 \\
\hline & $3 \mathrm{dxz}$ & 15.1 & $3 \mathrm{dxz}$ & 8.5 & $3 \mathrm{dxz}$ & 5.5 & $3 \mathrm{dxz}$ & 5.1 \\
\hline & $3 \mathrm{dyz}$ & 5.7 & $3 \mathrm{dyz}$ & 10.3 & 3dyz & 12.0 & 3dyz & 14.3 \\
\hline & $3 \mathrm{~d} x 2 \mathrm{y} 2$ & 1.4 & $3 \mathrm{~d} \times 2 \mathrm{y} 2$ & 0.7 & $3 \mathrm{~d} \times 2 \mathrm{y} 2$ & 0.7 & $3 \mathrm{~d} \times 2 \mathrm{y} 2$ & 0.3 \\
\hline & $3 \mathrm{dxy}$ & 0.2 & $3 \mathrm{dxy}$ & 0.2 & $3 \mathrm{dxy}$ & 0.7 & $3 \mathrm{dxy}$ & 0.5 \\
\hline & $4 \mathrm{dxz}$ & 5.8 & $4 \mathrm{dz} 2$ & 0.5 & $4 \mathrm{dz} 2$ & 0.7 & $4 \mathrm{dz} 2$ & 0.4 \\
\hline & $4 \mathrm{dyz}$ & 2.1 & $4 \mathrm{dxz}$ & 3.2 & $4 \mathrm{dxz}$ & 2.1 & $4 \mathrm{dxz}$ & 2.0 \\
\hline & $4 d x 2 y 2$ & 0.5 & 4dyz & 3.9 & 4dyz & 4.6 & 4dyz & 5.4 \\
\hline & $4 \mathrm{dxy}$ & 0.1 & $4 \mathrm{~d} \times 2 \mathrm{y} 2$ & 0.3 & $4 \mathrm{~d} \times 2 \mathrm{y} 2$ & 0.3 & $4 \mathrm{~d} \times 2 \mathrm{y} 2$ & 0.1 \\
\hline & $5 \mathrm{dxz}$ & 0.6 & $5 \mathrm{dxz}$ & 0.3 & $4 \mathrm{dxy}$ & 0.3 & $4 \mathrm{dxy}$ & 0.2 \\
\hline & $5 \mathrm{dyz}$ & 0.3 & 5 dyz & 0.6 & $5 \mathrm{dz} 2$ & 0.1 & $5 \mathrm{dxz}$ & 0.2 \\
\hline & & & & & $5 \mathrm{dxz}$ & 0.2 & 5 dyz & 0.8 \\
\hline & & & & & $5 \mathrm{dyz}$ & 0.7 & & \\
\hline & & & & & $5 \mathrm{dxy}$ & 0.1 & & \\
\hline & SUM & 31.7 & SUM & 29.8 & SUM & 29.7 & SUM & 30.4 \\
\hline \multirow{6}{*}{ 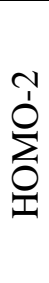 } & $4 \mathrm{pz}$ & 0.1 & $5 s$ & 0.2 & $4 \mathrm{pz}$ & 0.1 & $5 \mathrm{~s}$ & 0.1 \\
\hline & $5 \mathrm{pz}$ & 0.3 & $6 s$ & 0.2 & $5 \mathrm{pz}$ & 0.2 & $6 s$ & 0.1 \\
\hline & $3 \mathrm{dz} 2$ & 4.5 & $5 \mathrm{pz}$ & 0.2 & $3 \mathrm{dz} 2$ & 3.8 & $5 \mathrm{pz}$ & 0.2 \\
\hline & $3 \mathrm{dxz}$ & 5.9 & $3 \mathrm{dz} 2$ & 4.7 & $3 \mathrm{dxz}$ & 10.1 & $3 \mathrm{dz} 2$ & 3.9 \\
\hline & $3 \mathrm{dyz}$ & 14.4 & $3 \mathrm{dxz}$ & 8.0 & $3 \mathrm{dyz}$ & 10.4 & $3 \mathrm{dxz}$ & 11.6 \\
\hline & $3 \mathrm{dx} 2 \mathrm{y} 2$ & 0.2 & 3dyz & 14.9 & $3 \mathrm{~d} x 2 \mathrm{y} 2$ & 1.0 & 3dyz & 10.9 \\
\hline
\end{tabular}




\begin{tabular}{|c|c|c|c|c|c|c|c|c|}
\hline & $3 d x y$ & 2.4 & $3 \mathrm{~d} \times 2 \mathrm{y} 2$ & 0.1 & $3 \mathrm{dxy}$ & 0.8 & $3 \mathrm{~d} \times 2 \mathrm{y} 2$ & 0.6 \\
\hline & $4 \mathrm{dz} 2$ & 1.8 & $3 \mathrm{dxy}$ & 0.8 & $4 \mathrm{dz} 2$ & 1.6 & $3 \mathrm{dxy}$ & 0.5 \\
\hline & $4 \mathrm{dxz}$ & 2.5 & $4 \mathrm{dz} 2$ & 2.0 & $4 \mathrm{dxz}$ & 4.1 & $4 \mathrm{dz} 2$ & 1.6 \\
\hline & $4 \mathrm{dyz}$ & 5.8 & $4 \mathrm{dxz}$ & 3.2 & $4 \mathrm{dyz}$ & 4.2 & $4 \mathrm{dxz}$ & 4.7 \\
\hline & $4 \mathrm{dx} 2 \mathrm{y} 2$ & 0.1 & $4 d y z$ & 6.0 & $4 \mathrm{~d} \times 2 \mathrm{y} 2$ & 0.4 & $4 \mathrm{dyz}$ & 4.4 \\
\hline & $4 \mathrm{dxy}$ & 0.9 & $4 \mathrm{dx} 2 \mathrm{y} 2$ & 0.1 & $4 \mathrm{dxy}$ & 0.3 & $4 \mathrm{~d} \times 2 \mathrm{y} 2$ & 0.3 \\
\hline & $5 \mathrm{dz} 2$ & 0.2 & $4 \mathrm{dxy}$ & 0.3 & $5 \mathrm{dz} 2$ & 0.2 & $4 \mathrm{dxy}$ & 0.2 \\
\hline & $5 \mathrm{dxz}$ & 0.3 & $5 \mathrm{dz} 2$ & 0.4 & $5 \mathrm{dxz}$ & 0.6 & $5 \mathrm{dz} 2$ & 0.3 \\
\hline & $5 \mathrm{dyz}$ & 0.9 & $5 \mathrm{dxz}$ & 0.4 & $5 \mathrm{dyz}$ & 0.6 & $5 \mathrm{dxz}$ & 0.6 \\
\hline & $5 \mathrm{dxy}$ & 0.2 & $5 \mathrm{dyz}$ & 0.9 & $5 \mathrm{dxy}$ & 0.1 & $5 \mathrm{dyz}$ & 0.7 \\
\hline & & & $5 \mathrm{dxy}$ & 0.1 & & & & \\
\hline & SUM & 40.5 & SUM & 42.5 & SUM & 38.5 & SUM & 40.7 \\
\hline \multirow{22}{*}{$\sum_{\substack{0 \\
0}}^{\hat{0}}$} & $2 \mathrm{~s}$ & -0.6 & $2 \mathrm{~s}$ & -0.6 & $2 \mathrm{~s}$ & -0.5 & $2 \mathrm{~s}$ & -0.5 \\
\hline & $3 \mathrm{~s}$ & 1.3 & $3 \mathrm{~s}$ & 1.3 & $3 \mathrm{~s}$ & 1.3 & $3 \mathrm{~s}$ & 1.3 \\
\hline & $4 s$ & -0.8 & $4 s$ & -0.8 & $4 s$ & -0.8 & $4 s$ & -0.8 \\
\hline & $5 s$ & 13.8 & $5 s$ & 13.8 & $5 s$ & 13.6 & $5 s$ & 13.5 \\
\hline & $6 s$ & 10.7 & $6 s$ & 10.8 & $6 s$ & 10.2 & $6 s$ & 10.5 \\
\hline & $4 p x$ & 0.1 & $4 p x$ & 0.1 & $3 \mathrm{dz} 2$ & 31.8 & $3 \mathrm{dz} 2$ & 33.9 \\
\hline & $3 \mathrm{dz} 2$ & 33.2 & $3 \mathrm{dz} 2$ & 31.9 & $3 \mathrm{dxz}$ & 10.1 & $3 \mathrm{dxz}$ & 8.0 \\
\hline & $3 \mathrm{dxz}$ & 1.1 & $3 \mathrm{dxz}$ & 7.3 & $3 \mathrm{~d} x 2 \mathrm{y} 2$ & 1.7 & $3 \mathrm{dyz}$ & 0.1 \\
\hline & $3 \mathrm{dyz}$ & 6.6 & $3 \mathrm{dyz}$ & 0.6 & $3 \mathrm{~d} x y$ & 0.5 & $3 \mathrm{~d} \times 2 \mathrm{y} 2$ & 1.3 \\
\hline & $3 \mathrm{~d} x 2 \mathrm{y} 2$ & 0.2 & $3 \mathrm{~d} \times 2 \mathrm{y} 2$ & 1.5 & $4 \mathrm{dz} 2$ & 14.1 & $3 \mathrm{dxy}$ & 0.6 \\
\hline & $3 \mathrm{dxy}$ & 1.5 & $3 \mathrm{dxy}$ & 1.2 & $4 \mathrm{dxz}$ & 4.5 & $4 \mathrm{dz} 2$ & 15.1 \\
\hline & $4 \mathrm{dz} 2$ & 14.8 & $4 \mathrm{dz} 2$ & 14.3 & $4 \mathrm{dx} 2 \mathrm{y} 2$ & 0.6 & $4 \mathrm{dxz}$ & 3.6 \\
\hline & $4 \mathrm{dxz}$ & 0.5 & $4 \mathrm{dxz}$ & 3.3 & $4 \mathrm{dxy}$ & 0.2 & $4 \mathrm{~d} \times 2 \mathrm{y} 2$ & 0.5 \\
\hline & $4 \mathrm{dyz}$ & 2.9 & 4dyz & 0.3 & $5 \mathrm{dz} 2$ & 3.9 & $4 \mathrm{dxy}$ & 0.2 \\
\hline & $4 \mathrm{dxy}$ & 0.6 & $4 \mathrm{dx} 2 \mathrm{y} 2$ & 0.5 & $5 \mathrm{dxz}$ & 1.3 & $5 \mathrm{dz} 2$ & 4.2 \\
\hline & $5 \mathrm{dz} 2$ & 4.2 & $4 \mathrm{dxy}$ & 0.5 & $5 \mathrm{~d} \times 2 \mathrm{y} 2$ & 0.1 & $5 \mathrm{dxz}$ & 0.9 \\
\hline & $5 \mathrm{dxz}$ & 0.2 & $5 \mathrm{dz} 2$ & 4.1 & & & $5 \mathrm{~d} \times 2 \mathrm{y} 2$ & 0.1 \\
\hline & 5 dyz & 0.8 & $5 \mathrm{dxz}$ & 0.9 & & & & \\
\hline & $5 \mathrm{dxy}$ & 0.1 & $5 \mathrm{dyz}$ & 0.1 & & & & \\
\hline & & & $5 \mathrm{~d} \times 2 \mathrm{y} 2$ & 0.1 & & & & \\
\hline & & & $5 \mathrm{dxy}$ & 0.1 & & & & \\
\hline & SUM & 91.2 & SUM & 91.3 & SUM & 92.6 & SUM & 92.5 \\
\hline \multirow{10}{*}{ 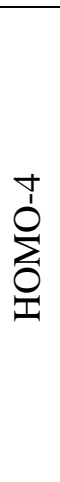 } & $3 \mathrm{dz} 2$ & 0.1 & $3 \mathrm{dz2}$ & 0.4 & $3 \mathrm{dz2}$ & 0.6 & $6 s$ & 0.1 \\
\hline & $3 \mathrm{dxz}$ & 2.3 & $3 \mathrm{dxz}$ & 2.0 & $3 \mathrm{dxz}$ & 1.3 & $3 \mathrm{dz} 2$ & 1.0 \\
\hline & 3dyz & 0.3 & $3 \mathrm{dyz}$ & 0.2 & 3dyz & 0.1 & $3 \mathrm{dxz}$ & 1.1 \\
\hline & $3 \mathrm{dxy}$ & 0.3 & $3 \mathrm{~d} \times 2 \mathrm{y} 2$ & 0.2 & $3 \mathrm{~d} \times 2 \mathrm{y} 2$ & 0.1 & $3 \mathrm{dyz}$ & 0.1 \\
\hline & $4 \mathrm{dxz}$ & 1.0 & $4 \mathrm{dz} 2$ & 0.2 & $4 \mathrm{dz} 2$ & 0.3 & $3 \mathrm{~d} \times 2 \mathrm{y} 2$ & 0.1 \\
\hline & $4 \mathrm{dyz}$ & 0.2 & $4 \mathrm{dxz}$ & 0.9 & $4 \mathrm{dxz}$ & 0.5 & $4 \mathrm{dz} 2$ & 0.5 \\
\hline & $4 d x y$ & 0.1 & 4dyz & 0.1 & 4dyz & 0.1 & $4 \mathrm{dxz}$ & 0.5 \\
\hline & $5 \mathrm{dxz}$ & 0.1 & $4 \mathrm{dx} 2 \mathrm{y} 2$ & 0.1 & $5 \mathrm{dxz}$ & 0.1 & $5 \mathrm{dz} 2$ & 0.1 \\
\hline & & & $5 \mathrm{dxz}$ & 0.1 & & & & \\
\hline & SUM & 4.4 & SUM & 4.2 & SUM & 3.1 & SUM & 3.5 \\
\hline
\end{tabular}


Table S6. Calculated formation of excited states of the complexes.

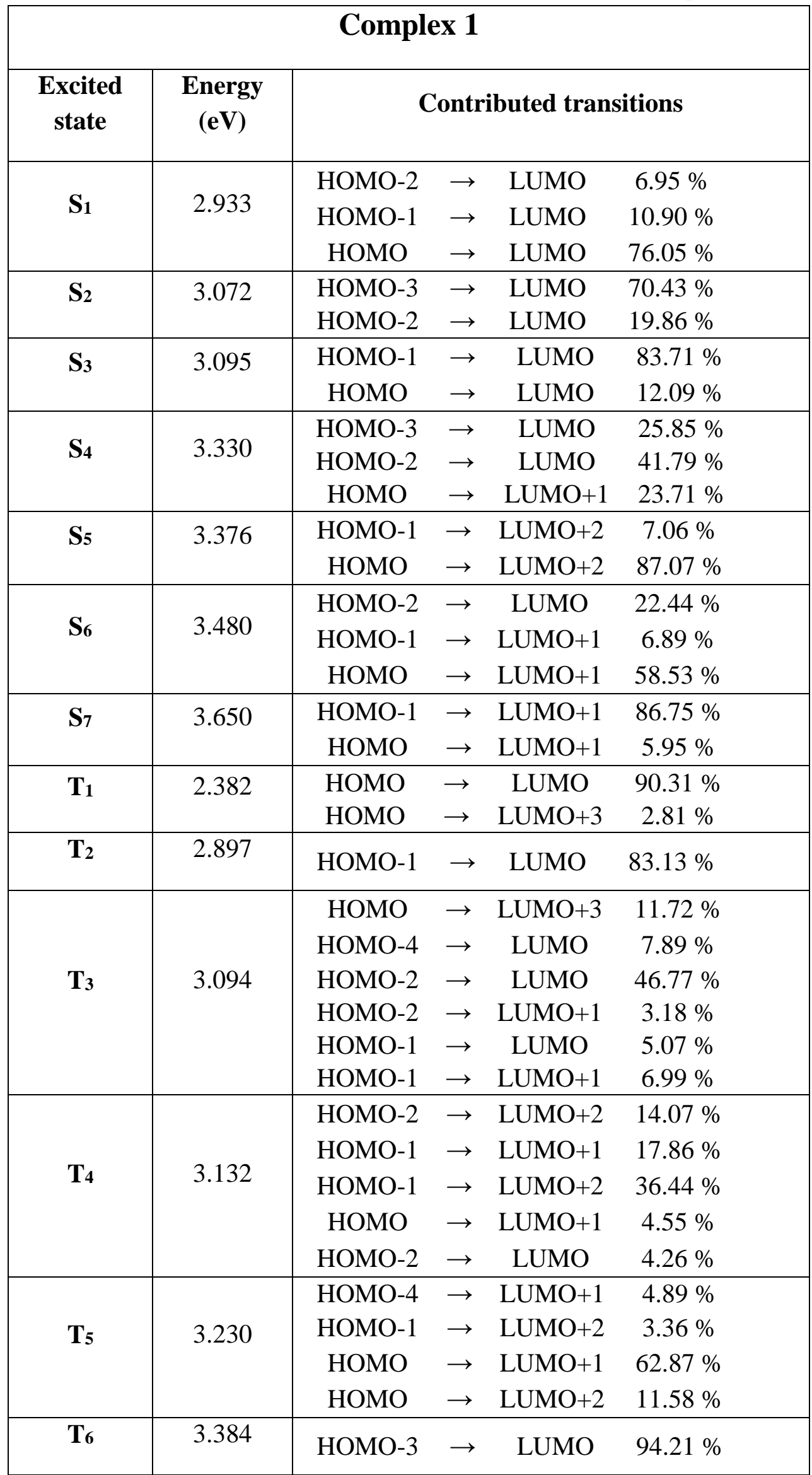




\begin{tabular}{|c|c|cccc|}
\hline \multirow{2}{*}{$\mathbf{T}_{7}$} & \multirow{3}{*}{3.412} & $\begin{array}{c}\text { HOMO-4 } \\
\text { HOMO-2 }\end{array}$ & $\rightarrow$ & LUMO & $33.38 \%$ \\
& & HOMO & $\rightarrow$ & LUMO+3 & $26.46 \%$ \\
\hline \multirow{3}{*}{$\mathbf{T}_{8}$} & \multirow{3}{*}{3.650} & HOMO-2 & $\rightarrow$ & LUMO+1 & $5.90 \%$ \\
& & HOMO-2 & $\rightarrow$ & LUMO+2 & $11.20 \%$ \\
& & HOMO & $\rightarrow$ & LUMO+1 & $12.10 \%$ \\
& & HOMO & $\rightarrow$ & LUMO+2 & $60.52 \%$ \\
\hline
\end{tabular}

\begin{tabular}{|c|c|c|c|c|c|}
\hline \multicolumn{6}{|c|}{ Complex 2} \\
\hline \multirow{4}{*}{$\begin{array}{c}\text { Excited } \\
\text { state }\end{array}$} & \multirow{4}{*}{$\begin{array}{c}\begin{array}{c}\text { Energy } \\
(\mathrm{eV})\end{array} \\
3.030\end{array}$} & \multicolumn{4}{|c|}{ Contributed transitions } \\
\hline & & HOMO-2 & $\rightarrow$ & LUMO & $7.69 \%$ \\
\hline & & HOMO-1 & $\rightarrow$ & LUMO & $9.14 \%$ \\
\hline & & HOMO & $\rightarrow$ & LUMO & $77.70 \%$ \\
\hline \multirow[t]{2}{*}{$\mathbf{S}_{2}$} & \multirow[t]{2}{*}{3.219} & HOMO-1 & $\rightarrow$ & LUMO & $86.40 \%$ \\
\hline & & HOMO & $\rightarrow$ & LUMO & $8.10 \%$ \\
\hline \multirow[t]{2}{*}{$\mathbf{S}_{\mathbf{3}}$} & \multirow[t]{2}{*}{3.225} & HOMO-3 & $\rightarrow$ & LUMO & $53.85 \%$ \\
\hline & & HOMO-2 & $\rightarrow$ & LUMO & $38.11 \%$ \\
\hline \multirow{4}{*}{$\mathbf{S}_{4}$} & \multirow{4}{*}{3.399} & HOMO-3 & $\rightarrow$ & LUMO & $43.34 \%$ \\
\hline & & HOMO-2 & $\rightarrow$ & LUMO & $39.64 \%$ \\
\hline & & HOMO & $\rightarrow$ & LUMO & $5.15 \%$ \\
\hline & & HOMO & $\rightarrow$ & LUMO+1 & $5.23 \%$ \\
\hline \multirow{3}{*}{$\mathbf{S}_{5}$} & \multirow{3}{*}{3.411} & HOMO-2 & $\rightarrow$ & LUMO & $5.40 \%$ \\
\hline & & HOMO-1 & $\rightarrow$ & LUMO+1 & $11.47 \%$ \\
\hline & & HOMO & $\rightarrow$ & LUMO+1 & $77.34 \%$ \\
\hline \multirow{3}{*}{$\mathbf{S}_{6}$} & \multirow{3}{*}{3.589} & HOMO-3 & $\rightarrow$ & LUMO+1 & $88.00 \%$ \\
\hline & & HOMO-3 & $\rightarrow$ & LUMO+2 & $4.58 \%$ \\
\hline & & HOMO-2 & $\rightarrow$ & LUMO+1 & $5.23 \%$ \\
\hline \multirow{3}{*}{$\mathbf{S}_{7}$} & \multirow{3}{*}{3.673} & HOMO-1 & $\rightarrow$ & LUMO+1 & $75.55 \%$ \\
\hline & & HOMO & $\rightarrow$ & LUMO+1 & $10.80 \%$ \\
\hline & & HOMO & $\rightarrow$ & LUMO+2 & $8.05 \%$ \\
\hline $\mathbf{T}_{\mathbf{1}}$ & 2.504 & HOMO & $\rightarrow$ & LUMO & $89.96 \%$ \\
\hline $\mathbf{T}_{2}$ & 2.972 & HOMO-1 & $\rightarrow$ & LUMO & $83.14 \%$ \\
\hline \multirow{5}{*}{$\mathbf{T}_{3}$} & \multirow{5}{*}{3.112} & HOMO-6 & $\rightarrow$ & LUMO+1 & $5.90 \%$ \\
\hline & & HOMO-1 & $\rightarrow$ & LUMO+1 & $52.88 \%$ \\
\hline & & HOMO-1 & $\rightarrow$ & LUMO+2 & $5.41 \%$ \\
\hline & & HOMO-2 & $\rightarrow$ & LUMO & $5.40 \%$ \\
\hline & & HOMO-2 & $\rightarrow$ & LUMO+1 & $13.34 \%$ \\
\hline \multirow{3}{*}{$\mathbf{T}_{4}$} & \multirow{3}{*}{3.171} & HOMO-1 & $\rightarrow$ & LUMO & $5.39 \%$ \\
\hline & & HOMO-4 & $\rightarrow$ & LUMO & $5.99 \%$ \\
\hline & & HOMO-2 & $\rightarrow$ & LUMO & $56.60 \%$ \\
\hline
\end{tabular}




\begin{tabular}{|c|c|cccc|}
\hline & & HOMO & $\rightarrow$ & LUMO+4 & $9.08 \%$ \\
\hline \multirow{5}{*}{$\mathbf{T}_{5}$} & \multirow{4}{*}{3.449} & HOMO-4 & $\rightarrow$ & LUMO & $11.39 \%$ \\
& & HOMO-3 & $\rightarrow$ & LUMO & $40.73 \%$ \\
& & HOMO-2 & $\rightarrow$ & LUMO & $10.13 \%$ \\
& & HOMO & $\rightarrow$ & LUMO+1 & $5.87 \%$ \\
& & HOMO & $\rightarrow$ & LUMO+2 & $8.97 \%$ \\
& & HOMO & $\rightarrow$ & LUMO+4 & $9.52 \%$ \\
\hline \multirow{5}{*}{$\mathbf{T}_{6}$} & \multirow{4}{*}{3.463} & HOMO-4 & $\rightarrow$ & LUMO+2 & $5.65 \%$ \\
& & HOMO-3 & $\rightarrow$ & LUMO & $7.32 \%$ \\
& & HOMO & $\rightarrow$ & LUMO+1 & $27.40 \%$ \\
& & HOMO & $\rightarrow$ & LUMO+2 & $36.09 \%$ \\
$\mathbf{T}_{7}$ & \multirow{4}{*}{3.477} & HOMO & $\rightarrow$ & LUMO+4 & $1.88 \%$ \\
& & HOMO & $\rightarrow$ & LUMO+4 & $10.82 \%$ \\
& & HOMO-4 & $\rightarrow$ & LUMO & $19.71 \%$ \\
& & HOMO-3 & $\rightarrow$ & LUMO & $46.79 \%$ \\
\hline \multirow{2}{*}{$\mathbf{T}_{8}$} & \multirow{3}{*}{3.673} & HOMO-2 & $\rightarrow$ & LUMO & $8.34 \%$ \\
& & HOMO & $\rightarrow$ & LUMO+1 & $38.53 \%$ \\
& & HOMO-2 & $\rightarrow$ & LUMO+1 & $21.10 \%$ \\
& & HOMO & $\rightarrow$ & LUMO+2 & $27.74 \%$ \\
\hline
\end{tabular}

\begin{tabular}{|c|c|c|c|c|c|}
\hline \multicolumn{6}{|c|}{ Complex 3} \\
\hline Excited & Energy & & Cont & ibuted tran & itions \\
\hline $\mathbf{S}_{1}$ & 2.757 & HOMO & $\rightarrow$ & LUMO & $86.93 \%$ \\
\hline $\mathbf{S}_{2}$ & 3.009 & HOMO-3 & $\rightarrow$ & LUMO & $96.85 \%$ \\
\hline $\mathbf{S}_{\mathbf{3}}$ & 3.164 & $\begin{array}{c}\text { HOMO-2 } \\
\text { HOMO-1 } \\
\text { HOMO }\end{array}$ & $\begin{array}{l}\rightarrow \\
\rightarrow \\
\rightarrow\end{array}$ & $\begin{array}{c}\text { LUMO } \\
\text { LUMO } \\
\text { LUMO+1 }\end{array}$ & $\begin{array}{l}6.04 \% \\
5.04 \% \\
82.50 \% \\
\end{array}$ \\
\hline $\mathbf{S}_{4}$ & 3.185 & HOMO & $\rightarrow$ & LUMO+2 & $89.64 \%$ \\
\hline $\mathbf{S}_{5}$ & 3.240 & $\begin{array}{c}\text { HOMO-1 } \\
\text { HOMO }\end{array}$ & $\begin{array}{l}\rightarrow \\
\rightarrow\end{array}$ & $\begin{array}{c}\text { LUMO } \\
\text { LUMO+1 }\end{array}$ & $\begin{array}{c}89.17 \% \\
5.39 \%\end{array}$ \\
\hline $\mathbf{S}_{6}$ & 3.340 & HOMO & $\rightarrow$ & LUMO+4 & $91.05 \%$ \\
\hline $\mathbf{S}_{7}$ & 3.483 & HOMO-2 & $\rightarrow$ & LUMO & $76.14 \%$ \\
\hline $\mathbf{T}_{1}$ & 1.939 & HOMO & $\rightarrow$ & LUMO & $94.45 \%$ \\
\hline $\mathbf{T}_{2}$ & 2.791 & $\begin{array}{c}\text { HOMO-2 } \\
\text { HOMO-1 } \\
\text { HOMO } \\
\text { HOMO } \\
\text { HOMO }\end{array}$ & $\begin{array}{l}\rightarrow \\
\rightarrow \\
\rightarrow \\
\rightarrow \\
\rightarrow\end{array}$ & $\begin{array}{c}\text { LUMO } \\
\text { LUMO } \\
\text { LUMO+1 } \\
\text { LUMO+2 } \\
\text { LUMO+3 }\end{array}$ & $\begin{array}{c}9.24 \% \\
19.09 \% \\
35.02 \% \\
23.53 \% \\
5.32 \%\end{array}$ \\
\hline
\end{tabular}




\begin{tabular}{|c|c|c|c|c|c|}
\hline $\mathbf{T}_{\mathbf{3}}$ & 2.976 & $\begin{array}{c}\text { HOMO-1 } \\
\text { HOMO } \\
\text { HOMO }\end{array}$ & $\begin{array}{l}\rightarrow \\
\rightarrow \\
\rightarrow\end{array}$ & $\begin{array}{c}\text { LUMO } \\
\text { LUMO+1 } \\
\text { LUMO+2 }\end{array}$ & $\begin{array}{c}57.29 \% \\
24.11 \% \\
5.15 \% \\
\end{array}$ \\
\hline $\mathbf{T}_{4}$ & 3.091 & $\begin{array}{c}\text { HOMO-2 } \\
\text { HOMO-4 } \\
\text { HOMO-1 } \\
\text { HOMO-1 } \\
\text { HOMO } \\
\text { HOMO } \\
\text { HOMO }\end{array}$ & $\begin{array}{l}\rightarrow \\
\rightarrow \\
\rightarrow \\
\rightarrow \\
\rightarrow \\
\rightarrow \\
\rightarrow\end{array}$ & $\begin{array}{c}\text { LUMO } \\
\text { LUMO } \\
\text { LUMO } \\
\text { LUMO+1 } \\
\text { LUMO+1 } \\
\text { LUMO+2 } \\
\text { LUMO+3 }\end{array}$ & $\begin{array}{c}15.86 \% \\
6.62 \% \\
9.17 \% \\
17.55 \% \\
12.47 \% \\
5.63 \% \\
13.92 \%\end{array}$ \\
\hline $\mathbf{T}_{\mathbf{5}}$ & 3.152 & $\begin{array}{c}\text { HOMO-1 } \\
\text { HOMO-1 } \\
\text { HOMO } \\
\text { HOMO } \\
\text { HOMO-2 }\end{array}$ & $\begin{array}{l}\rightarrow \\
\rightarrow \\
\rightarrow \\
\rightarrow \\
\rightarrow\end{array}$ & $\begin{array}{c}\text { LUMO+1 } \\
\text { LUMO+2 } \\
\text { LUMO+2 } \\
\text { LUMO+3 } \\
\text { LUMO }\end{array}$ & $\begin{array}{c}17.53 \% \\
26.93 \% \\
5.98 \% \\
10.60 \% \\
10.24 \%\end{array}$ \\
\hline $\mathbf{T}_{6}$ & 3.359 & $\begin{array}{c}\text { HOMO-4 } \\
\text { HOMO-2 } \\
\text { HOMO } \\
\text { HOMO } \\
\text { HOMO }\end{array}$ & $\begin{array}{l}\rightarrow \\
\rightarrow \\
\rightarrow \\
\rightarrow \\
\rightarrow\end{array}$ & $\begin{array}{c}\text { LUMO } \\
\text { LUMO } \\
\text { LUMO+1 } \\
\text { LUMO+2 } \\
\text { LUMO+3 }\end{array}$ & $\begin{array}{c}6.51 \% \\
36.02 \% \\
9.34 \% \\
24.99 \% \\
6.15 \%\end{array}$ \\
\hline $\mathbf{T}_{7}$ & 3.388 & HOMO-3 & $\rightarrow$ & LUMO & $94.44 \%$ \\
\hline $\mathbf{T}_{8}$ & 3.408 & $\begin{array}{c}\text { HOMO } \\
\text { HOMO } \\
\text { HOMO } \\
\text { HOMO-2 } \\
\text { HOMO-4 } \\
\text { HOMO-2 }\end{array}$ & $\begin{array}{l}\rightarrow \\
\rightarrow \\
\rightarrow \\
\rightarrow \\
\rightarrow\end{array}$ & $\begin{array}{c}\text { LUMO+1 } \\
\text { LUMO+2 } \\
\text { LUMO+3 } \\
\text { LUMO } \\
\text { LUMO } \\
\text { LUMO+2 }\end{array}$ & $\begin{array}{c}12.27 \% \\
27.48 \% \\
18.73 \% \\
12.27 \% \\
10.17 \% \\
6.71 \%\end{array}$ \\
\hline
\end{tabular}

\begin{tabular}{|c|c|c|c|c|c|}
\hline \multicolumn{6}{|c|}{ Complex 4} \\
\hline $\begin{array}{c}\text { Excited } \\
\text { state }\end{array}$ & $\begin{array}{c}\text { Energy } \\
(\mathrm{eV})\end{array}$ & & ont & ibuted tran & itions \\
\hline $\mathbf{S}_{1}$ & 2.810 & HOMO & $\rightarrow$ & LUMO & $89.20 \%$ \\
\hline $\mathbf{S}_{2}$ & 3.031 & $\begin{array}{c}\text { HOMO } \\
\text { HOMO-1 }\end{array}$ & $\begin{array}{l}\rightarrow \\
\rightarrow\end{array}$ & $\begin{array}{c}\text { LUMO+1 } \\
\text { LUMO }\end{array}$ & $\begin{array}{c}85.54 \% \\
8.52 \%\end{array}$ \\
\hline $\mathbf{S}_{\mathbf{3}}$ & 3.096 & HOMO-3 & $\rightarrow$ & LUMO & $93.82 \%$ \\
\hline $\mathbf{S}_{4}$ & 3.241 & HOMO & $\rightarrow$ & LUMO+4 & $93.74 \%$ \\
\hline S5 & 3.327 & $\begin{array}{c}\text { HOMO-1 } \\
\text { HOMO }\end{array}$ & $\rightarrow$ & $\begin{array}{c}\text { LUMO } \\
\text { LUMO+1 }\end{array}$ & $\begin{array}{l}83.28 \% \\
10.15 \%\end{array}$ \\
\hline $\mathbf{S}_{6}$ & 3.489 & $\begin{array}{c}\text { HOMO } \\
\text { HOMO-2 }\end{array}$ & $\begin{array}{l}\rightarrow \\
\rightarrow\end{array}$ & $\begin{array}{c}\text { LUMO+2 } \\
\text { LUMO }\end{array}$ & $\begin{array}{l}53.94 \% \\
36.68 \% \\
\end{array}$ \\
\hline
\end{tabular}




\begin{tabular}{|c|c|c|c|c|c|}
\hline $\mathbf{S}_{7}$ & 3.573 & $\begin{array}{c}\text { HOMO- } 2 \\
\text { HOMO } \\
\text { HOMO } \\
\text { HOMO }\end{array}$ & $\begin{array}{l}\rightarrow \\
\rightarrow \\
\rightarrow\end{array}$ & $\begin{array}{c}\text { LUMO } \\
\text { LUMO } \\
\text { LUMO+2 } \\
\text { LUMO+3 }\end{array}$ & $\begin{array}{c}47.83 \% \\
5.53 \% \\
30.73 \% \\
5.56 \% \\
\end{array}$ \\
\hline $\mathbf{T}_{1}$ & 1.974 & HOMO & $\rightarrow$ & LUMO & $94.48 \%$ \\
\hline $\mathbf{T}_{2}$ & 2.875 & $\begin{array}{c}\text { HOMO } \\
\text { HOMO-2 } \\
\text { HOMO-1 } \\
\text { HOMO }\end{array}$ & $\begin{array}{l}\rightarrow \\
\rightarrow \\
\rightarrow\end{array}$ & $\begin{array}{c}\text { LUMO+2 } \\
\text { LUMO } \\
\text { LUMO } \\
\text { LUMO+3 }\end{array}$ & $\begin{array}{c}30.18 \% \\
14.67 \% \\
35.39 \% \\
5.68 \%\end{array}$ \\
\hline $\mathbf{T}_{3}$ & 3.067 & $\begin{array}{c}\text { HOMO-1 } \\
\text { HOMO-4 } \\
\text { HOMO-1 } \\
\text { HOMO } \\
\text { HOMO }\end{array}$ & $\begin{array}{l}\rightarrow \\
\rightarrow \\
\rightarrow \\
\rightarrow\end{array}$ & $\begin{array}{c}\text { LUMO } \\
\text { LUMO } \\
\text { LUMO } \\
\text { LUMO+1 } \\
\text { LUMO+2 }\end{array}$ & $\begin{array}{c}50.95 \% \\
6.08 \% \\
50.95 \% \\
9.52 \% \\
20.26 \%\end{array}$ \\
\hline $\mathbf{T}_{4}$ & 3.093 & $\begin{array}{c}\text { HOMO-1 } \\
\text { HOMO } \\
\text { HOMO } \\
\text { HOMO-2 }\end{array}$ & $\begin{array}{l}\rightarrow \\
\rightarrow \\
\rightarrow \\
\rightarrow\end{array}$ & $\begin{array}{c}\text { LUMO+1 } \\
\text { LUMO+1 } \\
\text { LUMO+3 } \\
\text { LUMO }\end{array}$ & $\begin{array}{c}40.47 \% \\
28.99 \% \\
4.20 \% \\
5.66 \%\end{array}$ \\
\hline $\mathbf{T}_{5}$ & 3.196 & $\begin{array}{c}\text { HOMO-2 } \\
\text { HOMO-2 } \\
\text { HOMO-1 } \\
\text { HOMO-1 } \\
\text { HOMO } \\
\text { HOMO } \\
\text { HOMO }\end{array}$ & $\begin{array}{l}\rightarrow \\
\rightarrow \\
\rightarrow \\
\rightarrow \\
\rightarrow \\
\rightarrow\end{array}$ & $\begin{array}{c}\text { LUMO } \\
\text { LUMO+1 } \\
\text { LUMO+1 } \\
\text { LUMO+2 } \\
\text { LUMO+1 } \\
\text { LUMO+2 } \\
\text { LUMO+3 }\end{array}$ & $\begin{array}{c}14.75 \% \\
4.35 \% \\
17.64 \% \\
6.41 \% \\
10.07 \% \\
14.32 \% \\
15.29 \%\end{array}$ \\
\hline $\mathbf{T}_{6}$ & 3.352 & $\begin{array}{c}\text { HOMO-4 } \\
\text { HOMO-2 } \\
\text { HOMO-2 } \\
\text { HOMO-1 } \\
\text { HOMO } \\
\text { HOMO } \\
\text { HOMO }\end{array}$ & $\begin{array}{l}\rightarrow \\
\rightarrow \\
\rightarrow \\
\rightarrow \\
\rightarrow \\
\rightarrow \\
\rightarrow\end{array}$ & $\begin{array}{c}\text { LUMO } \\
\text { LUMO } \\
\text { LUMO+1 } \\
\text { LUMO+1 } \\
\text { LUMO+1 } \\
\text { LUMO+2 } \\
\text { LUMO+3 }\end{array}$ & $\begin{array}{c}5.94 \% \\
38.83 \% \\
4.57 \% \\
4.62 \% \\
18.75 \% \\
14.34 \% \\
4.58 \% \\
\end{array}$ \\
\hline $\mathbf{T}_{7}$ & 3.420 & $\begin{array}{c}\text { HOMO-4 } \\
\text { HOMO-2 } \\
\text { HOMO-2 } \\
\text { HOMO } \\
\text { HOMO } \\
\text { HOMO }\end{array}$ & $\begin{array}{l}\rightarrow \\
\rightarrow \\
\rightarrow \\
\rightarrow \\
\rightarrow \\
\rightarrow\end{array}$ & $\begin{array}{c}\text { LUMO } \\
\text { LUMO } \\
\text { LUMO+1 } \\
\text { LUMO+1 } \\
\text { LUMO+2 } \\
\text { LUMO+3 }\end{array}$ & $\begin{array}{c}8.75 \% \\
8.77 \% \\
4.25 \% \\
23.04 \% \\
10.89 \% \\
27.96 \% \\
\end{array}$ \\
\hline $\mathbf{T}_{8}$ & 3.440 & HOMO-3 & $\rightarrow$ & LUMO & $94.40 \%$ \\
\hline
\end{tabular}

\section{REFERENCES}

1. CrysAlisPro Software System, Agilent Technologies UK Ltd, Yarnton, Oxford, UK, 2014.

2. Dolomanov O.V., Bourhis L.J., Gildea R.J., Howard J.A.K., Puschmann H., J. Appl. Cryst., 2009, 42, 339. 
3. Sheldrick, G.M., Acta Cryst., 2008, A64, 339.

4. Neese F.,Wiley Interdiscip. Rev.: Comput. Mol. Sci., 2012, $2,73$.

5. Flores-Moreno R., Alvares-Mendez R. J., Vela A., and Köster A.M., J. Comput. Chem., 2006, $27,1009$.

6. Becke A.D., J. Chem. Phys., 1993, 98, 1372.

7. Sinnecker S., Rajendran A., Klamt A., Diedenhofen M., Neese F., J. Phys. Chem. 2006, 110, 2235.

8. F. Neese, F. Wennmohs, A. Hansen, and U. Becker. Chem. Phys., 2009, 356, 98. 